\title{
Star-galaxy separation by far-infrared color-color diagrams for the AKARI FIS all-sky survey (bright source catalog version $\beta-1)^{\star, \star \star}$
}

\author{
A. Pollo ${ }^{1,2}$, P. Rybka ${ }^{2}$, and T. T. Takeuchi ${ }^{3}$ \\ 1 The Andrzej Sołtan Institute for Nuclear Studies, ul. Hoża 69, 00-681 Warsaw, Poland \\ e-mail: apollo@fuw.edu.pl \\ 2 The Astronomical Observatory of the Jagiellonian University, ul. Orla 171, 30-244 Kraków, Poland \\ 3 Institute for Advanced Research, Nagoya University, Furo-cho, Chikusa-ku, Nagoya 464-8601, Japan
}

Received 8 October 2009 / Accepted 1 March 2010

\section{ABSTRACT}

\begin{abstract}
Aims. To separate stars and galaxies in the far infrared AKARI all-sky survey data, we have selected a sample with the complete color information available in the low extinction regions of the sky and constructed color-color plots for these data. We looked for the method of separating stars and galaxies using the color information.

Methods. We performed an extensive search for the counterparts of these selected all-sky survey objects in the NED and SIMBAD databases. Among 5176 selected objects, we found 4272 galaxies, 382 other extragalactic objects, 349 Milky Way stars, 50 other Galactic objects, and 101 sources detected before in various wavelengths but of an unknown origin. Twenty-two sources were left unidentified. Then, we checked the colors of stars and galaxies in the far-infrared flux-color and color-color plots.

Results. In the resulting diagrams, stars form two clearly separated clouds. One of them is easily distinguished from galaxies and allows for a simple method of excluding a large part of stars using the far-infrared data. The other smaller branch, overplotting galaxies, consists of stars known to have an infrared excess, like Vega and some fainter stars discovered by IRAS or 2MASS. The color properties of these objects in any case make them very difficult to distinguish from galaxies.

Conclusions. We conclude that the FIR color-color diagrams allow for a high-quality star-galaxy separation. With the proposed simple method we can select more that $95 \%$ of galaxies rejecting at least $80 \%$ of stars.
\end{abstract}

Key words. surveys - infrared: galaxies - dust, extinction - galaxies: fundamental parameters - infrared: stars

\section{Introduction}

Emission from galaxies at wavelengths beyond a few micrometers is produced mainly by dust. As widely known, star formation activity is always accompanied by dust production, though the actual mechanism of dust supply has not been very well understood yet. The energy emitted by massive stars at ultraviolet (UV) is scattered efficiently and finally absorbed by dust grains. The heated grains re-emit the energy at far-infrared (FIR). Thus, radiative processes in FIR are related to the composition and the amount of dust in galaxies, as well as to the properties of their stellar population, especially newly formed massive stars. Many studies have shown that a significant amount of star formation in galaxies is obscured by dust (Le Floc'h et al. 2005; Caputi et al. 2007; Buat et al. 2007a,b, 2008; Reddy et al. 2008). Especially, such "hidden" star formation is revealed as becoming more and more important with increasing redshift from $z=0$ to 1 (Takeuchi et al. 2005). Therefore, FIR observations are crucial for understanding the true star formation activity in the Universe.

\footnotetext{
* Appendices are only available in electronic form at http: //www . aanda.org

$\star \star$ Catalog is available in electronic form at the CDS via anonymous ftp to cdsarc.u-strasbg. fr (130.79.128.5) or via http://cdsweb.u-strasbg.fr/cgi-bin/qcat?J/A+A/514/A3
}

Any FIR surveys would first provide us with a point source catalog, and extragalactic astrophysicists may want to have a reliable list of galaxy candidates from the FIR catalog. However, it is usually not a trivial task to classify detected sources and extract a specific class of objects only from FIR information. Obviously, not only would galaxies be detected at FIR, but also many other kinds of Galactic objects would be included in a purely flux-limited catalog, which is obtained as a first product of the survey. Very crudely, one may cut out the Galactic plane to avoid Galactic star-forming regions or HII regions, but there are still numerous stars with dust, e.g., Vega-like stars and AGBs. An effective and convenient method of star-galaxy separation that makes use of FIR information only would be desired. Such a method would also be very useful for constructing a good candidate list of dusty stars.

IRAS (Neugebauer et al. 1984) has brought a vast amount of statistics, and very efficient methods have been invented from IRAS point source catalog (PSC). The four bands of IRAS enabled us even to perform a very detailed classification of extragalactic and various Galactic objects, such as blue and red galaxies, Seyferts and QSOs, carbon stars, HII regions, reflection nebulae, planetary nebulae, and T Tauri stars. A thorough description of the method from IRAS can be found in e.g., Walker et al. (1989).

After the many years since IRAS, the advent of AKARI (ASTRO-F) opened a new window to exploring the Universe, as 
a survey-oriented space telescope at mid-infrared (MIR) and FIR (Murakami et al. 2007). The primary purpose of the mission is to provide second-generation infrared (IR) catalogs to obtain better spatial resolution and wider spectral coverage than the IRAS catalog. AKARI is equipped with a cryogenically cooled telescope of $68.5 \mathrm{~cm}$ aperture diameter and two scientific instruments, the far-infrared surveyor (FIS; Kawada et al. 2007) and the infrared camera (IRC; Onaka et al. 2007). Among various astronomical observations performed by AKARI, an all-sky survey with FIS and IRC has been carried out (AKARI all-sky survey).

Since FIS is an instrument dedicated to FIR $\lambda=50-180 \mu \mathrm{m}$, all the AKARI FIS bands are in the FIR wavelengths: N60 $(65 \mu \mathrm{m}), W I D E-S(90 \mu \mathrm{m}), W I D E-L(140 \mu \mathrm{m})$, and N160 $(160 \mu \mathrm{m})$ (Kawada et al. 2007). Hereafter, we use a notation $S_{65}, S_{90}, S_{140}$, and $S_{160}$ for flux densities in these bands.

Especially, since FIS is sensitive at longer wavelengths than IRAS, a new method of classification is needed if we try to select a list of a certain class of objects. Such a scheme is not merely an empirical technique, but will also lead to a new understanding of objects with cool dust which were difficult to detect by IRAS bands.

Since our central interest is in the physics of IR galaxies, we set our main aim in selecting galaxies by fluxes at four AKARI FIS bands. In this paper, we present the first color-color and color-flux diagrams obtained by the all-sky survey data based only on FIS bands, and show the method of star-galaxy separation with these diagrams.

The paper is organized as follows: in Sect. 2, we present the data: the sample with the complete color information, In Sects. 3 and 4, we present the FIR flux-color and color-color diagrams of the data, respectively, and we show how galaxies and stars can be separated in them. We show our results and conclusions in Sect. 5.

\section{The data}

As mentioned in Introduction, one of the main missions of AKARI was to carry out the all-sky survey in the four photometric bands in the far-infrared wavelength ranging in $50-180 \mu \mathrm{m}$ with FIS (Kawada et al. 2007). The FIS scanned $94 \%$ of the entire sky more than twice in the 16 months of the cryogenic mission phase.

The AKARI FIS bright source catalog (hereafter BSC) is the first primary catalog from the AKARI all-sky survey. Data from the version $\beta-1$ of this catalog are used in this work. AKARI BSC is supposed to have a uniform detection limit, corresponding to per scan sensitivity, over the entire sky, except for very bright sky parts where different data acquisition mode had to be applied. Redundant observations were used to increase the reliability of the detection. A summary of the all-sky survey can be found in Yamamura et al. (2009).

The AKARI FIS BSC provides data for 64311 sources, among them 43342 sources with fluxes measured at all the four FIR bands. For each detected source, AKARI source identifier, equatorial coordinates of the source position and flux densities in the four FIR bands are given. The position accuracy of the FIS BSC is $8^{\prime \prime}$, since the source extraction is made with grids of this size. Effective size of the point spread function of AKARI FIS in $F W H M$ is estimated to be $37 \pm 1^{\prime \prime}, 39 \pm 1,58 \pm 3^{\prime \prime}$, and $61 \pm 4^{\prime \prime}$ at N60, WIDE-S, WIDE-L, and N160, respectively (Kawada et al. 2007). Errors are not estimated for each individual source, but instead they are in total estimated to be $35 \%, 30 \%, 60 \%$, and $60 \%$ at N60, WIDE-S, WIDE-L, and N160, respectively (Yamamura et al. 2008).

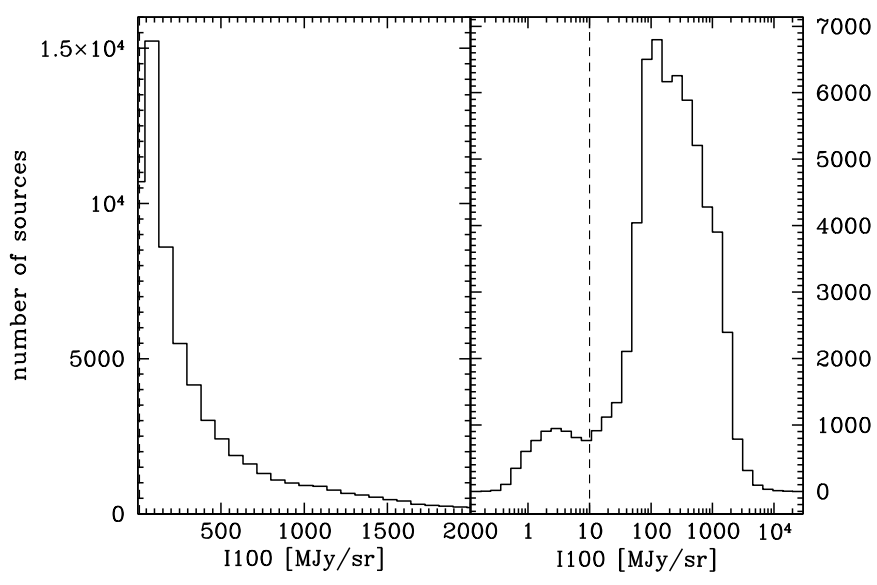

Fig. 1. Histogram of the number of objects as a function of the COBE DIRBE $100 \mu \mathrm{m}$ sky emission intensity at the position of each object, made from Schlegel et al. (1998). The vertical dashed line in the righthand panel represents the criterion we have adopted on the Galactic cirrus emission for this analysis $\left(I_{100}<10 \mathrm{MJy} \mathrm{sr}^{-1}\right)$.

The Galactic $100 \mu \mathrm{m}$ emission at each position was obtained from the so-called SFD maps (Schlegel et al. 1998). The distribution of the number of the sources as a function of the $100-\mu \mathrm{m}$ Galactic emission at the position of each source is shown in Fig. 1: left panel is the distribution in linear scale, and right panel is in logarithmic scale. For optical observations, as is widely known, heavily obscured regions of the sky are not ideal for source finding and photometry. Since the biggest available database is often found at $B$-band, the extinction would be large and may affect the probability of counterpart association, even if we can correct the extinction for detected objects. On the FIR side, dust grains in the Galactic interstellar medium emit thermal radiation at these wavelengths. The quality of flux measurement at high-Galactic foreground regions would be affected by the FIR diffuse Galactic emission, and sometimes it is difficult to remove the effect completely. Since both stem from the Galactic dust, putting a threshold on the extinction $E(B-V)$ and the diffuse foreground radiation would give almost equivalent results. Hence, we have selected objects lying in regions associated with low Galactic emission at $100 \mu \mathrm{m}$. We set our threshold to be $10 \mathrm{MJy} \mathrm{sr}^{-1}$, which is a very conservative value, but thanks to this, we could perform the subsequent analysis safely. This step left us with 6030 sources. The sky distribution of these sources is presented in Fig. 2. By this criterion, the selected sources naturally avoid the Galactic plane region. As a result, most of the Galactic sources, which are the majority of the AKARI FIS sources, are not included in our sample. This is seen in the lefthand panel of Fig. 1. The number of the sources in the selected regions is much smaller than that of the excluded regions. However, the area of the selected region is $77.5 \%$ of the total sky area.

Among selected sources, 5176 sources have full four-band information. These sources were then used to construct colorcolor and flux-color diagrams presented in the subsequent sections.

The next step was to identify these selected sources with known objects in public databases. For that purpose, we searched the NED and SIMBAD databases looking for the nearest counterparts within $40^{\prime \prime}$-circle around positions of AKARI sources. This diameter of $40^{\prime \prime}$ was chosen since the FWHM of AKARI FIS at WIDE-S is $39^{\prime \prime}$; i.e., this is large enough compared to the 


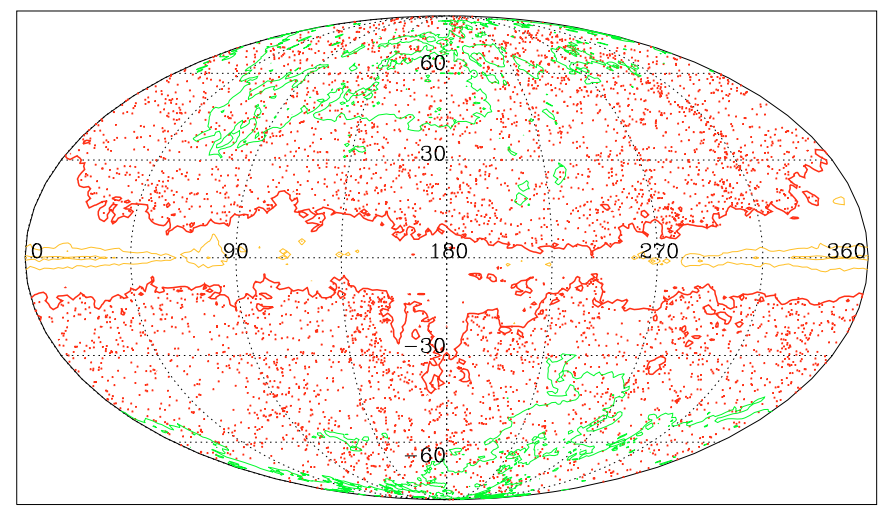

Fig. 2. The sky distribution of the AKARI FIS sources in the low FIR sky emissivity regions, overplotted with the COBE DIRBE $100 \mu \mathrm{m}$ sky emission intensity $I_{100}$. Contour levels are logarithmically spaced from $I_{100}=10^{-1}, 10^{0}, 10^{1}, 10^{2}, 10^{3}$, and $10^{4} \mathrm{MJy} \mathrm{sr}^{-1}$. The thick contours indicate our criterion $I_{100}<10 \mathrm{MJy} \mathrm{sr}^{-1}$.

Table 1. Classification of 5176 sources with a complete photometry located in the sky regions of low $100 \mu \mathrm{m}$ emissivity $\left(I_{100}<10 \mathrm{MJy} \mathrm{sr}^{-1}\right)$.

\begin{tabular}{|c|c|c|}
\hline Galaxies & & 4272 \\
\hline \multirow[t]{10}{*}{ Other extragalactic objects } & & 382 \\
\hline & pairs of galaxies & 65 \\
\hline & triplets of galaxies & 4 \\
\hline & groups of galaxies & 6 \\
\hline & parts of galaxies & 50 \\
\hline & stars in nearby galaxies & 148 \\
\hline & HII regions & 76 \\
\hline & supernovae hosts & 28 \\
\hline & quasi-stellar objects & 2 \\
\hline & emission-line objects & 2 \\
\hline \multirow{4}{*}{ Milky Way stars } & & 349 \\
\hline & variable stars & 264 \\
\hline & peculiar stars & 38 \\
\hline & other stars & 47 \\
\hline \multirow[t]{8}{*}{ Other galactic objects } & & 50 \\
\hline & associations of stars & 2 \\
\hline & planetary nebulae & 35 \\
\hline & reflection nebulae & 2 \\
\hline & other nebulae & 1 \\
\hline & Galactic cirruses & 5 \\
\hline & HII regions & 1 \\
\hline & OH masers & 4 \\
\hline Other sources & & 101 \\
\hline radio sources & & 33 \\
\hline infrared sources & & 48 \\
\hline X-ray sources & & 20 \\
\hline Unidentified sources & & 22 \\
\hline
\end{tabular}

position accuracy of AKARI $\left(8^{\prime \prime}\right)$ and small enough not to have too many interlopers. The summary of the identified sources is given in Table 1. In total, 399 Galactic and 4654 extragalactic objects were identified. In addition, we found 101 sources observed in other wavelengths for which it is not clear whether they are of Galactic or extragalactic origin. Only 22 sources were left unidentified.

Though we do not have direct information on the redshift distribution of the sample, there are related studies that can give an idea: Takeuchi et al. (2010) associate the FIS BSC with SDSS and IRAS PSC $z$ and show the redshift distribution of the
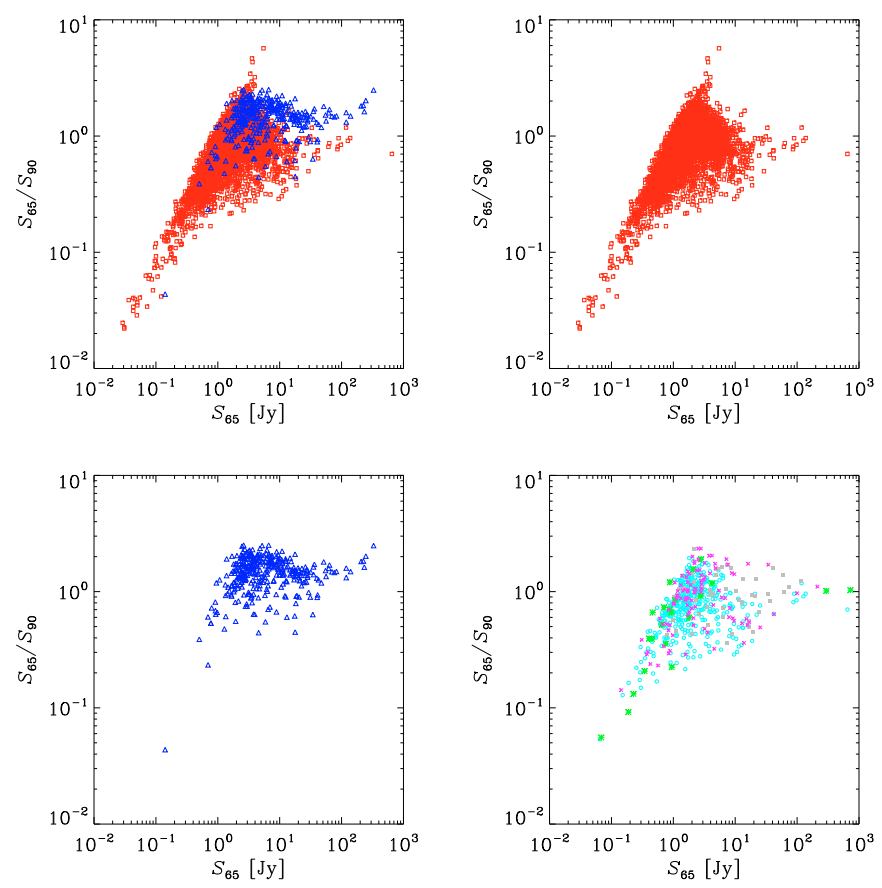

Fig. 3. The $S_{65}-S_{65} / S_{90}$ flux-color diagram. Top-left: galaxies and stars, top-right: galaxies, bottom-left: stars, and bottom-right: remaining objects: Galactic, extragalactic, those with identifications but without information whether they are Galactic or extragalactic, or ones that were unidentified. Symbols represent the sources as follows: open squares (red in the onlnie version): galaxies; open triangles (blue): the Galactic stars; open circles (cyan): other extragalactic objects (QSOs, stars in extragalactic objects); filled squares (gray): other Galactic objects (star associations, nebulae, HII regions, cirruses); asterisks (green): unknown origin; crosses (magenta): sources without counterparts.

subsample of BSC with ultraviolet, optical, NIR, and FIR counterparts. They find that the vast majority of their sample are located at $z<0.05$ (see their Fig. 3). Małek et al. (2010) made a counterpart association of the $90-\mu \mathrm{m}$ sources detected in the deep FIS survey data in the AKARI deep field south. The fraction of redshift association was not sufficiently high, but Małek et al. (2010) find that most of bright galaxies $\left(S_{90}>100 \mathrm{mJy}\right)$ are at $z \leq 0.01$. These studies suggest that most of our sample is located at very low redshifts. Hence, we can safely ignore any cosmological effects such as $K$-correction, since it is much smaller than the uncertainties in flux measurements.

For further studies, however, a possible effect of redshift may become significant. Since our sample is selected by FIS WIDE-S $(90 \mu \mathrm{m})$, it is dominated by galaxies with relatively cool dust. Hence, if we had a high-redshift galaxy in this sample, it would appear to be redder on the flux-color or color-color diagrams. Then, in the diagrams we discuss in this paper, such a galaxy would be even farther from stars than nearby galaxies. However, the effect is not very drastic because the FIS does not have submillimeter bands. The redshift effect was previously discussed by Takeuchi et al. (1999) as a way to find high-redshift galaxies from the FIS color-color diagrams (see their Fig. 9).

\section{Flux-color diagrams}

Two examples of flux-color diagrams constructed from four FIS measurements are presented in Figs. 3 and 4. Since other 

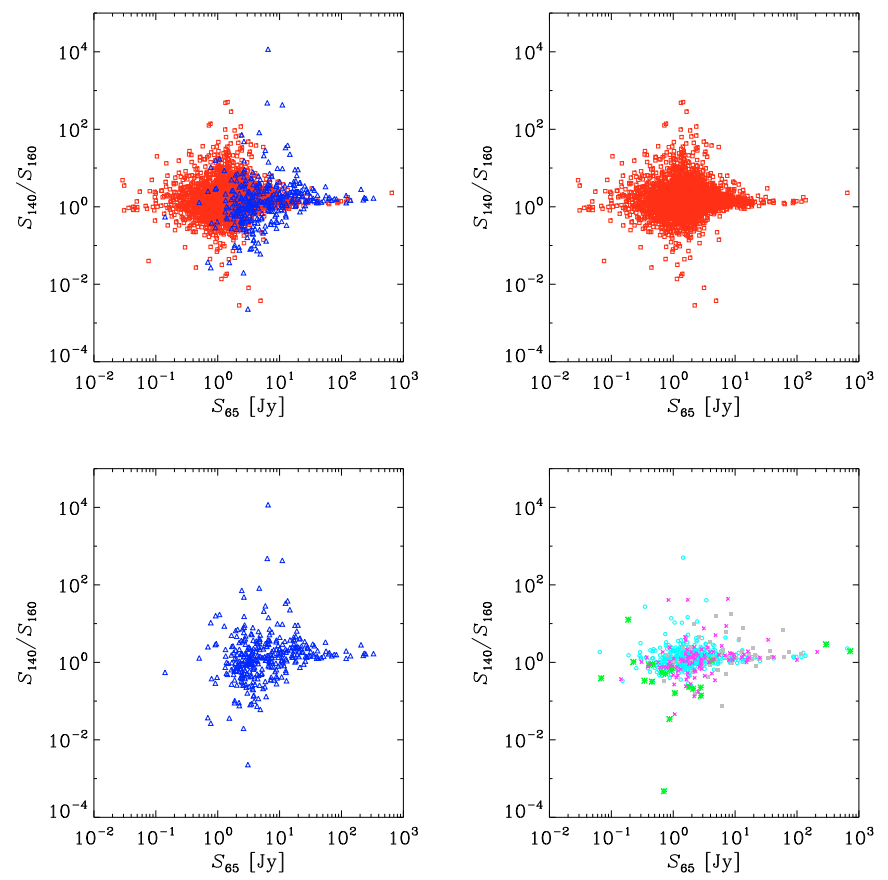

Fig. 4. Same as Fig. 3 but for $S_{65}-S_{140} / S_{160}$.

Table 2. Division lines between stars and galaxies for color-color diagrams.

\begin{tabular}{llrr}
\hline \hline Color 1 & Color 2 & $a$ & $b$ \\
\hline$S_{65} / S_{90}$ & $S_{65} / S_{140}$ & -0.17 & 0.33 \\
$S_{65} / S_{90}$ & $S_{65} / S_{160}$ & -4.08 & 1.18 \\
$S_{65} / S_{90}$ & $S_{90} / S_{140}$ & -1.24 & 0.32 \\
$S_{65} / S_{90}$ & $S_{90} / S_{160}$ & -4.08 & 0.97 \\
$S_{65} / S_{90}$ & $S_{140} / S_{160}$ & -49.09 & 10.01 \\
$S_{65} / S_{140}$ & $S_{65} / S_{160}$ & -2.95 & 1.39 \\
$S_{65} / S_{140}$ & $S_{90} / S_{140}$ & -2.18 & 1.20 \\
$S_{65} / S_{140}$ & $S_{90} / S_{160}$ & -2.46 & 1.00 \\
$S_{65} / S_{140}$ & $S_{140} / S_{160}$ & -1.81 & 0.60 \\
$S_{65} / S_{160}$ & $S_{90} / S_{140}$ & -0.46 & 0.40 \\
$S_{65} / S_{160}$ & $S_{90} / S_{160}$ & 2.36 & -1.04 \\
$S_{65} / S_{160}$ & $S_{140} / S_{160}$ & 1.31 & -0.49 \\
$S_{90} / S_{140}$ & $S_{90} / S_{160}$ & -3.94 & 1.38 \\
$S_{90} / S_{140}$ & $S_{140} / S_{160}$ & -9.20 & 2.33 \\
$S_{90} / S_{160}$ & $S_{140} / S_{160}$ & 1.19 & -0.30 \\
\hline
\end{tabular}

diagrams contain very similar information, the remaining diagrams are presented only in electronic form, as online Figs. A.1A.11.

At the first glance, in the presented diagrams we clearly see two separate branches at brighter flux densities in Fig. 3: one branch is for galaxies, and the other is for stars. For Fig. 4, since both bands are longer than $100 \mu \mathrm{m}$, it is very possible that both are at the Rayleigh-Jeans regime of their dust emission. Consequently, we cannot expect a clear distinction between stars and galaxies because they are quite similar to each other.

For the other colors, the contiguous set of AKARI FIS bands (Kawada et al. 2007) enables us to grasp the peak of dust spectrum. This reflects the distinct two branches on the flux-color diagrams. Though stars show a slight double-peaked distribution, one branch is much more prominent than the second one. We revisit the issue of the identities of these stars and, in particular, the smaller, redder branch of stars, later.
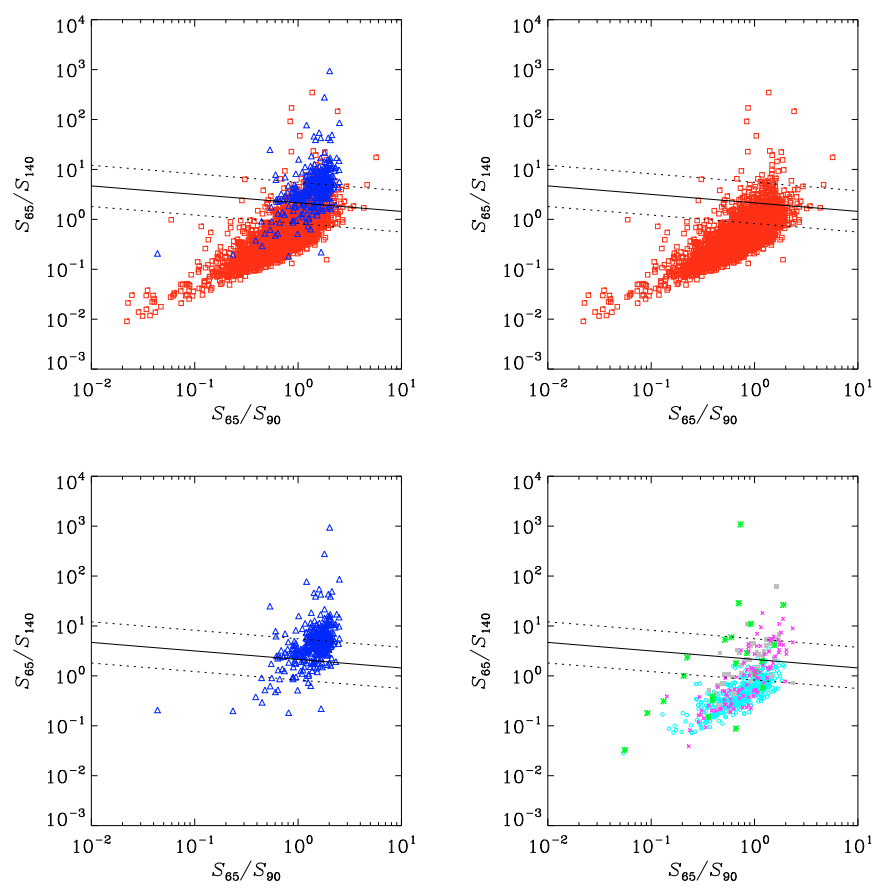

Fig. 5. The $S_{65} / S_{90}-S_{65} / S_{140}$ color-color diagrams of the sample. The format and symbols in the panels are the same as those in flux-color diagrams.
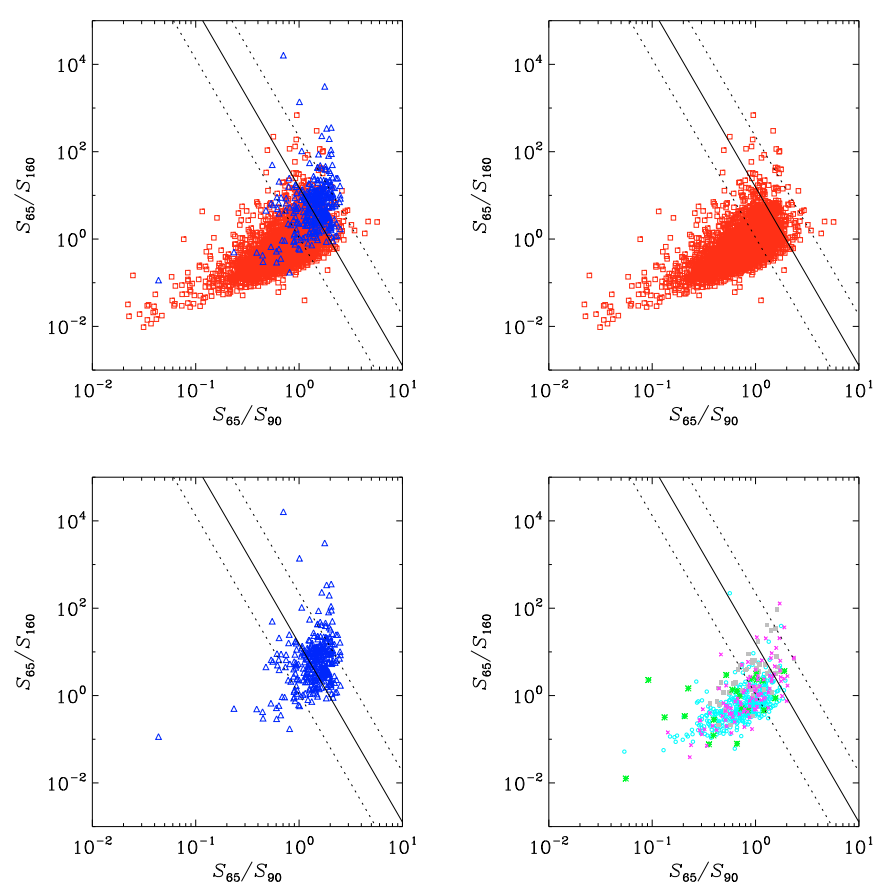

Fig. 6. Same as Fig. 5 but for $S_{65} / S_{90}-S_{65} / S_{160}$.

In sum, these diagrams encourage us to go further to explore the classification scheme from the AKARI FIS bands.

\section{Color-color diagrams}

We show the color-color diagrams constructed from four FIS measurements in Figs. 5-8. Again, we do not show all, but four among 15 independent diagrams. The rest of them are presented only in an electronic form as online Figs. B.1-B.6. 

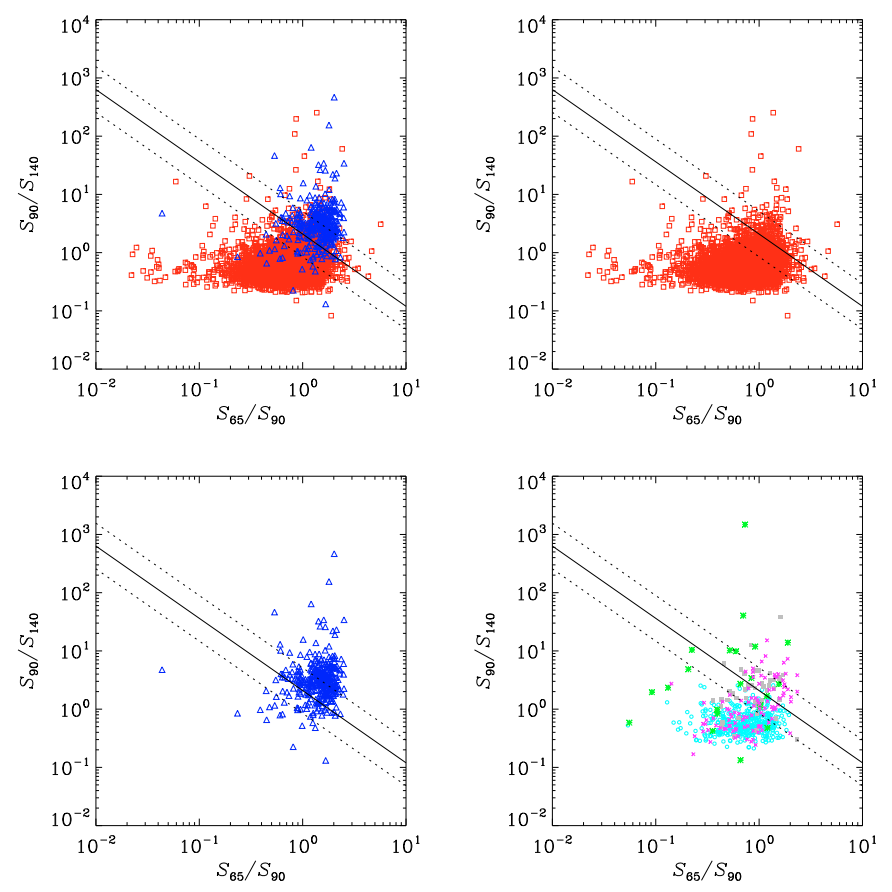

Fig. 7. Same as Fig. 5 but for $S_{65} / S_{90}-S_{90} / S_{140}$.

Based on these scatter plots, we decided the division line between stars and galaxies. We did not try to make a detailed statistical discriminant analysis but to put them in by hand. One may worry that this procedure would be "subjective", but in the following examination we show that our determination works very well and is almost perfectly consistent. We plan to show a more statistically sophisticated analysis elsewhere.

The central solid straight line shown in each panel represents the best division boundary separating galaxies and stars. The parameters of these lines for each diagram, $a$ and $b$ in the following equation

Color $2=a \times($ Color 1$)+b$,

are listed in Table 2. The dotted lines represent the uncertainty ranges in the color determination corresponding to the average photometric errors: $35 \%, 30 \%, 60 \%$, and $60 \%$ at N60, WIDE$S$, WIDE-L, and N160, respectively. As shown in Table 3, in all cases the quality of separation is very good, especially for galaxies. To two sides of the dividing line, we refer there to as the galaxy cloud (the side dominated by galaxies) and the stellar cloud (the side dominated by stars). It is shown that galaxies are selected with accuracy ranging between $95 \%$ and $98 \%$. Stars, again, form two separate branches between which one, usually consisting of more than $80 \%$ of stars, is well separated and the second, smaller one overlaps galaxies.

The quality of the division is affected by the photometric errors, at least in case of some diagrams. As shown in Table 4, by considering only objects located in the diagrams outside the "error bands" between the lines corresponding to photometric errors, we obtain a much better separation of galaxies, always above $98 \%$, and often well above $99 \%$. In contrast, the quality of the separation of stars becomes less reliable. First, the number statistics of stars often becomes poor with such a selection. Second, we can observe a clear split between diagrams: in most cases the quality of star separation becomes better, above $90 \%$, which can prove that both our division and the error estimation are correct. However, in some cases (the ones with the least of stars left outside the error bands) the quality estimated in such
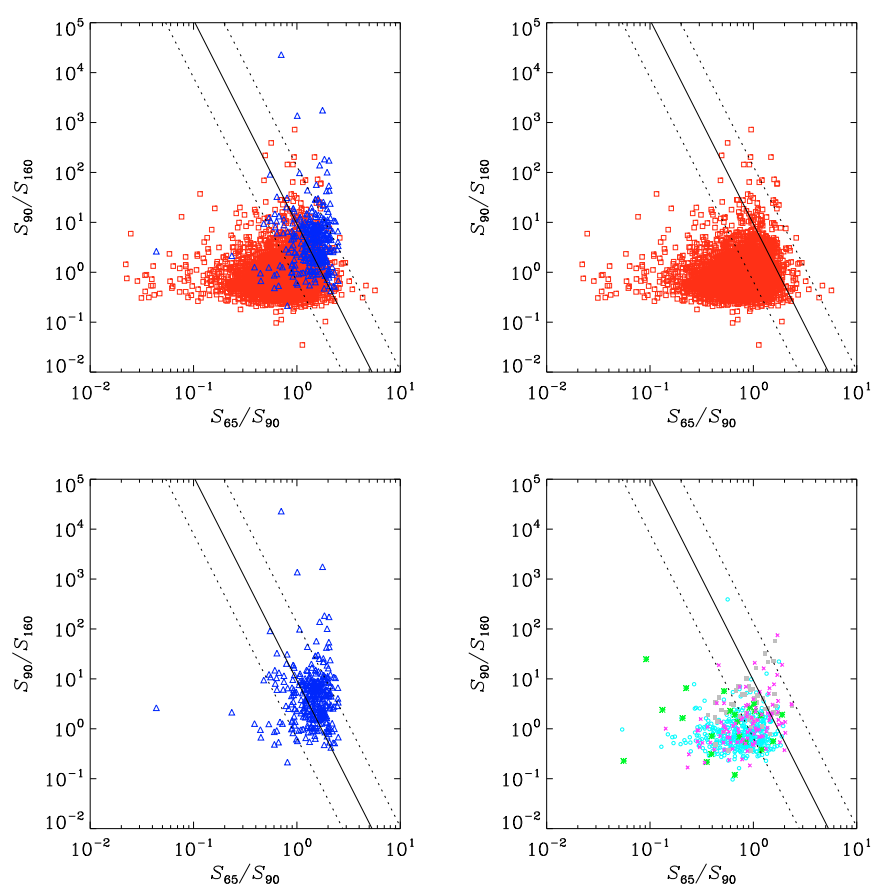

Fig. 8. Same as Fig. 5 but for $S_{65} / S_{90}-S_{90} / S_{160}$.

a way drops significantly. Apart from the statistical reason, this may be related to larger uncertainties in the measurement of the FIR fluxes, in particular $S_{160}$.

In this place it is also worth drawing the reader's attention to Figs. 5 and 6. The difference between them is the use of color along the $y$ axis: in Fig. 5 it is $S_{65} / S_{140}$ and in Fig. $6-S_{65} / S_{160}$. Given the small difference in wavelengths, we expect that for given objects these colors and the separation lines would be similar on the diagrams. However, the best division lines are significantly different from each other.

With the division lines applied, the quality of the galaxy separation is very good in both these diagrams, but the quality of star separation is significantly lower for Fig. 6. A plausible explanation, again, may be larger uncertainties than the assumed ones in the flux measurement at $N 160$ in the case of the $\beta-1$ version of the FIS PSC. It should be stressed, however, that even when taking this possibility into account, the quality of the separation in all the diagrams remains high.

As shown in Table 5, the consistency of the division is very high for selected galaxies (usually higher than 90\%). For stars, the consistency of the division varies much more strongly among flux-color diagrams, but in most cases the percentage of correct categorization is also very high.

We checked the properties of stars belonging to both groups. We used the separation obtained in the diagram $S_{65} / S_{90}$ vs. $S_{90} / S_{140}$, which is shown in Fig. 7. From the consistency checks we can assume that the diagnosis of these stars will be roughly applicable for most of the other diagrams as well.

A well-separated branch of stars in this diagram consists of 295 objects. Most of them are optically bright stars. This sample is dominated by variable stars, mainly Mira-type stars (35\%), and other pulsating stars $(40 \%)$. There are also a few binary systems. Among the rest we find evolved stars like carbon stars (6\%), stars with envelope of OH/IR type (4\%), and a few other AGB and post-AGB stars. We also find a few faint sources identified as stars by big surveys like SDSS. We call this branch of stars "Branch I". 
Table 3. The quality of star-galaxy separation in color-color diagrams.

\begin{tabular}{|c|c|c|c|c|c|c|c|c|}
\hline Diagram & \multicolumn{2}{|c|}{$\begin{array}{l}\text { Galaxies in the galaxy cloud } \\
\text { number percentage }\end{array}$} & \multicolumn{2}{|c|}{$\begin{array}{l}\text { Stars in the star cloud } \\
\text { number percentage }\end{array}$} & \multicolumn{2}{|c|}{$\begin{array}{l}\text { Galaxies in the star cloud } \\
\text { number percentage }\end{array}$} & \multicolumn{2}{|c|}{$\begin{array}{l}\text { Stars in the galaxy cloud } \\
\text { number percentage }\end{array}$} \\
\hline$S_{65} / S_{90}$ vs. $S_{65} / S_{140}$ & 4114 & 96.30 & 290 & 83.09 & 158 & 3.70 & 59 & 16.91 \\
\hline$S_{65} / S_{90}$ vs. $S_{65} / S_{160}$ & 4110 & 96.21 & 226 & 64.76 & 162 & 3.79 & 123 & 35.24 \\
\hline$S_{65} / S_{90}$ vs. $S_{90} / S_{140}$ & 4096 & 95.88 & 295 & 84.53 & 176 & 4.12 & 54 & 15.47 \\
\hline$S_{65} / S_{90}$ vs. $S_{90} / S_{160}$ & 4085 & 95.62 & 245 & 70.20 & 187 & 4.38 & 104 & 29.80 \\
\hline$S_{65} / S_{90}$ vs. $S_{140} / S_{160}$ & 4109 & 96.18 & 144 & 41.26 & 163 & 3.82 & 205 & 58.74 \\
\hline$S_{65} / S_{140}$ vs. $S_{65} / S_{160}$ & 4120 & 96.44 & 294 & 84.24 & 152 & 3.56 & 55 & 15.76 \\
\hline$S_{65} / S_{140}$ vs. $S_{90} / S_{140}$ & 4162 & 97.43 & 274 & 78.51 & 110 & 2.57 & 75 & 21.49 \\
\hline$S_{65} / S_{140}$ vs. $S_{90} / S_{160}$ & 4081 & 95.53 & 304 & 87.11 & 191 & 4.47 & 45 & 12.89 \\
\hline$S_{65} / S_{140}$ vs. $S_{140} / S_{160}$ & 4018 & 94.05 & 301 & 86.25 & 254 & 5.95 & 48 & 13.75 \\
\hline$S_{65} / S_{160}$ vs. $S_{90} / S_{140}$ & 4107 & 96.14 & 293 & 83.95 & 165 & 3.86 & 56 & 16.05 \\
\hline$S_{65} / S_{160}$ vs. $S_{90} / S_{160}$ & 4128 & 96.63 & 198 & 56.73 & 144 & 3.37 & 151 & 43.27 \\
\hline$S_{65} / S_{160}$ vs. $S_{140} / S_{160}$ & 4147 & 97.07 & 288 & 82.52 & 125 & 2.93 & 61 & 17.48 \\
\hline$S_{90} / S_{140}$ vs. $S_{90} / S_{160}$ & 4094 & 95.83 & 286 & 81.95 & 178 & 4.17 & 63 & 18.05 \\
\hline$S_{90} / S_{140}$ vs. $S_{140} / S_{160}$ & 4098 & 95.93 & 289 & 82.81 & 174 & 4.07 & 60 & 17.19 \\
\hline$S_{90} / S_{160}$ vs. $S_{140} / S_{160}$ & 4081 & 95.53 & 291 & 83.38 & 191 & 4.47 & 58 & 16.62 \\
\hline
\end{tabular}

Table 4. The quality of star-galaxy separation in color-color diagrams outside of the areas between the lines indicating photometric errors.

\begin{tabular}{|c|c|c|c|c|c|c|c|c|c|c|}
\hline \multirow[t]{2}{*}{ Diagram } & \multicolumn{2}{|c|}{ Total number } & \multicolumn{2}{|c|}{$\begin{array}{c}\text { Galaxies } \\
\text { in the galaxy cloud }\end{array}$} & \multicolumn{2}{|c|}{$\begin{array}{c}\text { Stars } \\
\text { in the star cloud }\end{array}$} & \multicolumn{2}{|c|}{$\begin{array}{c}\text { Galaxies } \\
\text { in the star cloud }\end{array}$} & \multicolumn{2}{|c|}{$\begin{array}{c}\text { Stars } \\
\text { in the galaxy cloud }\end{array}$} \\
\hline & galaxies & stars & number & percentage & number & percentage & number & percentage & number & percentage \\
\hline$S_{65} / S_{90}$ vs. $S_{65} / S_{140}$ & 3328 & 157 & 3297 & 99.07 & 142 & 90.45 & 31 & 0.93 & 15 & 9.55 \\
\hline$S_{65} / S_{90}$ vs. $S_{65} / S_{160}$ & 2989 & 67 & 2970 & 99.36 & 38 & 56.72 & 19 & 0.64 & 29 & 43.28 \\
\hline$S_{65} / S_{90}$ vs. $S_{90} / S_{140}$ & 3370 & 185 & 3335 & 98.96 & 168 & 90.81 & 35 & 1.04 & 17 & 9.19 \\
\hline$S_{65} / S_{90}$ vs. $S_{90} / S_{160}$ & 2583 & 52 & 2568 & 99.42 & 34 & 65.38 & 15 & 0.58 & 18 & 34.62 \\
\hline$S_{65} / S_{90}$ vs. $S_{140} / S_{160}$ & 2123 & 31 & 2118 & 99.76 & 0 & 0.00 & 5 & 0.24 & 31 & 100.00 \\
\hline$S_{65} / S_{140}$ vs. $S_{65} / S_{160}$ & 3631 & 207 & 3600 & 99.15 & 189 & 91.30 & 31 & 0.85 & 18 & 8.70 \\
\hline$S_{65} / S_{140}$ vs. $S_{90} / S_{140}$ & 3901 & 165 & 3865 & 99.08 & 138 & 83.64 & 36 & 0.92 & 27 & 16.36 \\
\hline$S_{65} / S_{140}$ vs. $S_{90} / S_{160}$ & 3491 & 226 & 3444 & 98.65 & 213 & 94.25 & 47 & 1.35 & 13 & 5.75 \\
\hline$S_{65} / S_{140}$ vs. $S_{140} / S_{160}$ & 2896 & 181 & 2855 & 98.58 & 170 & 93.92 & 41 & 1.42 & 11 & 6.08 \\
\hline$S_{65} / S_{160}$ vs. $S_{90} / S_{140}$ & 3525 & 201 & 3489 & 98.98 & 184 & 91.54 & 36 & 1.02 & 17 & 8.46 \\
\hline$S_{65} / S_{160}$ vs. $S_{90} / S_{160}$ & 3129 & 59 & 3111 & 99.42 & 37 & 62.71 & 18 & 0.58 & 22 & 37.29 \\
\hline$S_{65} / S_{160}$ vs. $S_{140} / S_{160}$ & 3353 & 142 & 3335 & 99.46 & 129 & 90.85 & 18 & 0.54 & 13 & 9.15 \\
\hline$S_{90} / S_{140}$ vs. $S_{90} / S_{160}$ & 3345 & 157 & 3308 & 98.89 & 142 & 90.45 & 37 & 1.11 & 15 & 9.55 \\
\hline$S_{90} / S_{140}$ vs. $S_{140} / S_{160}$ & 2787 & 99 & 2758 & 98.96 & 93 & 93.94 & 29 & 1.04 & 6 & 6.06 \\
\hline$S_{90} / S_{160}$ vs. $S_{140} / S_{160}$ & 2245 & 80 & 2223 & 99.02 & 76 & 95.00 & 22 & 0.98 & 4 & 5.00 \\
\hline
\end{tabular}

The second less prominent branch of stars, mixed with galaxies, consists of 54 objects. We call this branch "Branch II". A significant fraction of the objects in this branch are stars that were originally discovered in the infrared by IRAS or 2MASS (28\%) or other stars already known to have a significant excess of the infrared luminosity (the most notable is Vega). In addition, we again have a significant number of different types of pulsating variables $(32 \%)$ but this time only two Mira-type stars among them, five faint stars and three T Tauri stars.

It is also interesting to take a look at the distribution of the other Galactic sources. They are divided much more equally between two branches, and slightly more $(60 \%)$ overlaps for Branch II. All the masers, as well as $43 \%$ of planetary nebulae, are located in the area corresponding to Branch I. The remaining $57 \%$ of planetary nebulae, as well as all the other nebulae, HII regions, and all the Galactic cirruses overlap Branch II.

For that reason, if we divide not only just between stars and galaxies, but also between the galactic and extragalactic objects, the percentage of the Galactic objects in the galaxy cloud typically becomes a few percent higher.

As we see, stars and other Galactic objects that occupy the area of the "galaxy cloud" typically belong to special classes of extremely red sources and it may require much more sophisticated techniques than just simple one-line division in the colorcolor diagram to distinguish them from the extragalactic sources.

The division we presented and discussed here allows for an efficient separation of more than $95 \%$ of galaxies and around $80 \%$ of stars in the two parts of the diagram. As it was already mentioned before, some diagrams, even if still applicable, are obviously worse than the others, in particular when it comes to the separation of stars. A plausible reason are the larger uncertainties in the FIR flux measurement of the $\beta$ - 1 version of the FIS PSC, most probably in the longest wavelength filter N160. A more detailed classification of Galactic and extragalactic sources based on their color properties will be the subject of a subsequent paper.

Sources for which it is unknown whether their origin is Galactic or extragalactic in the diagrams appear on both sides of the dividing lines. While only about $10 \%$ of a total number of objects from our initial sample are located in the stellar part of the diagrams, the fraction in the stellar cloud rises to about $30 \%$ for these objects. One possible interpretation is that these objects are more often of a Galactic origin. However, they may also be extragalactic sources with extreme colors. 
A. Pollo et al.: Star-galaxy separation by far infrared colors

Table 5. Consistency of star and galaxy identifications between different color-color diagrams.

\begin{tabular}{|c|c|c|c|c|c|}
\hline Diagram 1 & Diagram 2 & $\begin{array}{c}\text { Galaxies } \\
\text { in the galaxy cloud } \\
{[\%]}\end{array}$ & $\begin{array}{c}\text { Stars } \\
\text { in the star cloud } \\
{[\%]}\end{array}$ & $\begin{array}{c}\text { Galaxies } \\
\text { in the star cloud } \\
{[\%]}\end{array}$ & $\begin{array}{c}\text { Stars } \\
\text { in the galaxy cloud } \\
{[\%]}\end{array}$ \\
\hline$S_{65} / S_{90}$ vs. $S_{65} / S_{140}$ & $S_{65} / S_{90}$ vs. $S_{65} / S_{160}$ & 97.23 & 82.17 & 28.75 & 49.45 \\
\hline$S_{65} / S_{90}$ vs. $S_{65} / S_{140}$ & $S_{65} / S_{90}$ vs. $S_{90} / S_{140}$ & 99.76 & 99.15 & 94.01 & 95.58 \\
\hline$S_{65} / S_{90}$ vs. $S_{65} / S_{140}$ & $S_{65} / S_{90}$ vs. $S_{90} / S_{160}$ & 96.99 & 85.23 & 28.41 & 51.53 \\
\hline$S_{65} / S_{90}$ vs. $S_{65} / S_{140}$ & $S_{65} / S_{90}$ vs. $S_{140} / S_{160}$ & 97.14 & 63.13 & 26.79 & 39.39 \\
\hline$S_{65} / S_{90}$ vs. $S_{65} / S_{140}$ & $S_{65} / S_{140}$ vs. $S_{65} / S_{160}$ & 98.86 & 95.89 & 69.68 & 78.95 \\
\hline$S_{65} / S_{90}$ vs. $S_{65} / S_{140}$ & $S_{65} / S_{140}$ vs. $S_{90} / S_{140}$ & 99.30 & 96.81 & 78.36 & 86.57 \\
\hline$S_{65} / S_{90}$ vs. $S_{65} / S_{140}$ & $S_{65} / S_{140}$ vs. $S_{90} / S_{160}$ & 98.57 & 95.62 & 66.48 & 75.00 \\
\hline$S_{65} / S_{90}$ vs. $S_{65} / S_{140}$ & $S_{65} / S_{140}$ vs. $S_{140} / S_{160}$ & 97.05 & 93.40 & 41.75 & 63.55 \\
\hline$S_{65} / S_{90}$ vs. $S_{65} / S_{140}$ & $S_{65} / S_{160}$ vs. $S_{90} / S_{140}$ & 98.48 & 95.37 & 61.30 & 76.52 \\
\hline$S_{65} / S_{90}$ vs. $S_{65} / S_{140}$ & $S_{65} / S_{160}$ vs. $S_{90} / S_{160}$ & 97.02 & 76.23 & 18.54 & 44.76 \\
\hline$S_{65} / S_{90}$ vs. $S_{65} / S_{140}$ & $S_{65} / S_{160}$ vs. $S_{140} / S_{160}$ & 99.00 & 95.50 & 70.67 & 78.33 \\
\hline$S_{65} / S_{90}$ vs. $S_{65} / S_{140}$ & $S_{90} / S_{140}$ vs. $S_{90} / S_{160}$ & 98.37 & 93.75 & 60.12 & 70.49 \\
\hline$S_{65} / S_{90}$ vs. $S_{65} / S_{140}$ & $S_{90} / S_{140}$ vs. $S_{140} / S_{160}$ & 98.66 & 94.30 & 66.87 & 72.27 \\
\hline$S_{65} / S_{90}$ vs. $S_{65} / S_{140}$ & $S_{90} / S_{160}$ vs. $S_{140} / S_{160}$ & 98.43 & 94.66 & 63.04 & 73.50 \\
\hline$S_{65} / S_{90}$ vs. $S_{65} / S_{160}$ & $S_{65} / S_{90}$ vs. $S_{90} / S_{140}$ & 97.22 & 82.53 & 32.54 & 48.59 \\
\hline$S_{65} / S_{90}$ vs. $S_{65} / S_{160}$ & $S_{65} / S_{90}$ vs. $S_{90} / S_{160}$ & 99.57 & 95.97 & 89.97 & 91.63 \\
\hline$S_{65} / S_{90}$ vs. $S_{65} / S_{160}$ & $S_{65} / S_{90}$ vs. $S_{140} / S_{160}$ & 98.33 & 77.30 & 57.85 & 74.39 \\
\hline$S_{65} / S_{90}$ vs. $S_{65} / S_{160}$ & $S_{65} / S_{140}$ vs. $S_{65} / S_{160}$ & 97.96 & 84.23 & 46.50 & 53.93 \\
\hline$S_{65} / S_{90}$ vs. $S_{65} / S_{160}$ & $S_{65} / S_{140}$ vs. $S_{90} / S_{140}$ & 97.44 & 80.40 & 22.06 & 50.51 \\
\hline$S_{65} / S_{90}$ vs. $S_{65} / S_{160}$ & $S_{65} / S_{140}$ vs. $S_{90} / S_{160}$ & 97.50 & 83.02 & 41.93 & 46.43 \\
\hline$S_{65} / S_{90}$ vs. $S_{65} / S_{160}$ & $S_{65} / S_{140}$ vs. $S_{140} / S_{160}$ & 97.71 & 84.25 & 55.29 & 51.46 \\
\hline$S_{65} / S_{90}$ vs. $S_{65} / S_{160}$ & $S_{65} / S_{160}$ vs. $S_{90} / S_{140}$ & 97.16 & 80.92 & 28.75 & 44.69 \\
\hline$S_{65} / S_{90}$ vs. $S_{65} / S_{160}$ & $S_{65} / S_{160}$ vs. $S_{90} / S_{160}$ & 98.64 & 82.55 & 63.40 & 72.99 \\
\hline$S_{65} / S_{90}$ vs. $S_{65} / S_{160}$ & $S_{65} / S_{160}$ vs. $S_{140} / S_{160}$ & 97.95 & 84.05 & 41.11 & 55.43 \\
\hline$S_{65} / S_{90}$ vs. $S_{65} / S_{160}$ & $S_{90} / S_{140}$ vs. $S_{90} / S_{160}$ & 96.78 & 79.30 & 22.35 & 43.01 \\
\hline$S_{65} / S_{90}$ vs. $S_{65} / S_{160}$ & $S_{90} / S_{140}$ vs. $S_{140} / S_{160}$ & 96.64 & 79.22 & 17.86 & 41.53 \\
\hline$S_{65} / S_{90}$ vs. $S_{65} / S_{160}$ & $S_{90} / S_{160}$ vs. $S_{140} / S_{160}$ & 96.59 & 79.69 & 20.96 & 41.99 \\
\hline$S_{65} / S_{90}$ vs. $S_{90} / S_{140}$ & $S_{65} / S_{90}$ vs. $S_{90} / S_{160}$ & 97.01 & 85.93 & 32.51 & 51.90 \\
\hline$S_{65} / S_{90}$ vs. $S_{90} / S_{140}$ & $S_{65} / S_{90}$ vs. $S_{140} / S_{160}$ & 97.14 & 63.33 & 30.68 & 37.84 \\
\hline$S_{65} / S_{90}$ vs. $S_{90} / S_{140}$ & $S_{65} / S_{140}$ vs. $S_{65} / S_{160}$ & 98.83 & 95.76 & 70.73 & 77.06 \\
\hline$S_{65} / S_{90}$ vs. $S_{90} / S_{140}$ & $S_{65} / S_{140}$ vs. $S_{90} / S_{140}$ & 99.06 & 95.96 & 72.73 & 82.17 \\
\hline$S_{65} / S_{90}$ vs. $S_{90} / S_{140}$ & $S_{65} / S_{140}$ vs. $S_{90} / S_{160}$ & 98.54 & 95.83 & 67.57 & 74.75 \\
\hline$S_{65} / S_{90}$ vs. $S_{90} / S_{140}$ & $S_{65} / S_{140}$ vs. $S_{140} / S_{160}$ & 97.07 & 93.62 & 44.65 & 62.75 \\
\hline$S_{65} / S_{90}$ vs. $S_{90} / S_{140}$ & $S_{65} / S_{160}$ vs. $S_{90} / S_{140}$ & 98.33 & 94.90 & 59.82 & 72.73 \\
\hline$S_{65} / S_{90}$ vs. $S_{90} / S_{140}$ & $S_{65} / S_{160}$ vs. $S_{90} / S_{160}$ & 96.96 & 76.27 & 21.88 & 42.93 \\
\hline$S_{65} / S_{90}$ vs. $S_{90} / S_{140}$ & $S_{65} / S_{160}$ vs. $S_{140} / S_{160}$ & 98.92 & 95.03 & 70.43 & 74.78 \\
\hline$S_{65} / S_{90}$ vs. $S_{90} / S_{140}$ & $S_{90} / S_{140}$ vs. $S_{90} / S_{160}$ & 98.19 & 93.29 & 58.19 & 66.67 \\
\hline$S_{65} / S_{90}$ vs. $S_{90} / S_{140}$ & $S_{90} / S_{140}$ vs. $S_{140} / S_{160}$ & 98.44 & 93.84 & 63.43 & 68.42 \\
\hline$S_{65} / S_{90}$ vs. $S_{90} / S_{140}$ & $S_{90} / S_{160}$ vs. $S_{140} / S_{160}$ & 98.28 & 94.20 & 61.58 & 69.64 \\
\hline$S_{65} / S_{90}$ vs. $S_{90} / S_{160}$ & $S_{65} / S_{90}$ vs. $S_{140} / S_{160}$ & 97.90 & 73.52 & 50.86 & 66.67 \\
\hline$S_{65} / S_{90}$ vs. $S_{90} / S_{160}$ & $S_{65} / S_{140}$ vs. $S_{65} / S_{160}$ & 97.79 & 87.20 & 46.61 & 56.60 \\
\hline$S_{65} / S_{90}$ vs. $S_{90} / S_{160}$ & $S_{65} / S_{140}$ vs. $S_{90} / S_{140}$ & 97.17 & 83.24 & 21.55 & 51.40 \\
\hline$S_{65} / S_{90}$ vs. $S_{90} / S_{160}$ & $S_{65} / S_{140}$ vs. $S_{90} / S_{160}$ & 97.43 & 86.70 & 44.44 & 51.01 \\
\hline$S_{65} / S_{90}$ vs. $S_{90} / S_{160}$ & $S_{65} / S_{140}$ vs. $S_{140} / S_{160}$ & 97.89 & 87.91 & 61.22 & 56.58 \\
\hline$S_{65} / S_{90}$ vs. $S_{90} / S_{160}$ & $S_{65} / S_{160}$ vs. $S_{90} / S_{140}$ & 97.05 & 84.01 & 31.25 & 46.25 \\
\hline$S_{65} / S_{90}$ vs. $S_{90} / S_{160}$ & $S_{65} / S_{160}$ vs. $S_{90} / S_{160}$ & 98.70 & 85.33 & 67.67 & 74.51 \\
\hline$S_{65} / S_{90}$ vs. $S_{90} / S_{160}$ & $S_{65} / S_{160}$ vs. $S_{140} / S_{160}$ & 97.76 & 87.05 & 41.03 & 58.18 \\
\hline$S_{65} / S_{90}$ vs. $S_{90} / S_{160}$ & $S_{90} / S_{140}$ vs. $S_{90} / S_{160}$ & 96.61 & 82.49 & 24.11 & 44.31 \\
\hline$S_{65} / S_{90}$ vs. $S_{90} / S_{160}$ & $S_{90} / S_{140}$ vs. $S_{140} / S_{160}$ & 96.42 & 82.40 & 18.84 & 42.68 \\
\hline$S_{65} / S_{90}$ vs. $S_{90} / S_{160}$ & $S_{90} / S_{160}$ vs. $S_{140} / S_{160}$ & 96.42 & 82.84 & 22.75 & 43.21 \\
\hline$S_{65} / S_{90}$ vs. $S_{140} / S_{160}$ & $S_{65} / S_{140}$ vs. $S_{65} / S_{160}$ & 97.29 & 63.01 & 29.21 & 37.69 \\
\hline$S_{65} / S_{90}$ vs. $S_{140} / S_{160}$ & $S_{65} / S_{140}$ vs. $S_{90} / S_{140}$ & 97.21 & 60.77 & 15.38 & 41.43 \\
\hline$S_{65} / S_{90}$ vs. $S_{140} / S_{160}$ & $S_{65} / S_{140}$ vs. $S_{90} / S_{160}$ & 96.73 & 61.61 & 24.29 & 31.20 \\
\hline$S_{65} / S_{90}$ vs. $S_{140} / S_{160}$ & $S_{65} / S_{140}$ vs. $S_{140} / S_{160}$ & 96.27 & 62.92 & 27.34 & 34.78 \\
\hline$S_{65} / S_{90}$ vs. $S_{140} / S_{160}$ & $S_{65} / S_{160}$ vs. $S_{90} / S_{140}$ & 96.52 & 59.50 & 12.80 & 32.18 \\
\hline$S_{65} / S_{90}$ vs. $S_{140} / S_{160}$ & $S_{65} / S_{160}$ vs. $S_{90} / S_{160}$ & 97.32 & 57.31 & 28.01 & 58.99 \\
\hline$S_{65} / S_{90}$ vs. $S_{140} / S_{160}$ & $S_{65} / S_{160}$ vs $S_{140} / S_{160}$ & 97.46 & 62.96 & 27.08 & 39.85 \\
\hline$S_{65} / S_{90}$ vs. $S_{140} / S_{160}$ & $S_{90} / S_{140}$ vs. $S_{90} / S_{160}$ & 96.23 & 57.67 & 9.38 & 32.09 \\
\hline$S_{65} / S_{90}$ vs. $S_{140} / S_{160}$ & $S_{90} / S_{140}$ vs $S_{140} / S_{160}$ & 96.21 & 57.74 & 7.72 & 30.94 \\
\hline$S_{65} / S_{90}$ vs. $S_{140} / S_{160}$ & $S_{90} / S_{160}$ vs. $S_{140} / S_{160}$ & 96.07 & 57.93 & 9.04 & 30.42 \\
\hline$S_{65} / S_{140}$ vs. $S_{65} / S_{160}$ & $S_{65} / S_{140}$ vs. $S_{90} / S_{140}$ & 98.96 & 95.07 & 67.18 & 78.46 \\
\hline$S_{65} / S_{140}$ vs. $S_{65} / S_{160}$ & $S_{65} / S_{140}$ vs. $S_{90} / S_{160}$ & 99.45 & 98.33 & 86.88 & 90.00 \\
\hline
\end{tabular}


Table 5. continued.

\begin{tabular}{|c|c|c|c|c|c|}
\hline Diagram 1 & Diagram 2 & $\begin{array}{c}\text { Galaxies } \\
\text { in the galaxy cloud } \\
{[\%]}\end{array}$ & $\begin{array}{c}\text { Stars } \\
\text { in the star cloud } \\
{[\%]}\end{array}$ & $\begin{array}{c}\text { Galaxies } \\
\text { in the star cloud } \\
{[\%]}\end{array}$ & $\begin{array}{c}\text { Stars } \\
\text { in the galaxy cloud } \\
{[\%]} \\
\end{array}$ \\
\hline$S_{65} / S_{140}$ vs. $S_{65} / S_{160}$ & $S_{65} / S_{140}$ vs. $S_{140} / S_{160}$ & 98.13 & 96.81 & 62.56 & 81.55 \\
\hline$S_{65} / S_{140}$ vs. $S_{65} / S_{160}$ & $S_{65} / S_{160}$ vs. $S_{90} / S_{140}$ & 98.99 & 97.10 & 73.82 & 84.68 \\
\hline$S_{65} / S_{140}$ vs. $S_{65} / S_{160}$ & $S_{65} / S_{160}$ vs. $S_{90} / S_{160}$ & 97.99 & 80.08 & 43.92 & 52.43 \\
\hline$S_{65} / S_{140}$ vs. $S_{65} / S_{160}$ & $S_{65} / S_{160}$ vs. $S_{140} / S_{160}$ & 99.67 & 98.97 & 90.25 & 94.83 \\
\hline$S_{65} / S_{140}$ vs. $S_{65} / S_{160}$ & $S_{90} / S_{140}$ vs. $S_{90} / S_{160}$ & 98.61 & 95.52 & 65.45 & 77.97 \\
\hline$S_{65} / S_{140}$ vs. $S_{65} / S_{160}$ & $S_{90} / S_{140}$ vs. $S_{140} / S_{160}$ & 98.52 & 95.71 & 62.58 & 78.26 \\
\hline$S_{65} / S_{140}$ vs. $S_{65} / S_{160}$ & $S_{90} / S_{160}$ vs. $S_{140} / S_{160}$ & 98.52 & 96.07 & 64.72 & 79.65 \\
\hline$S_{65} / S_{140}$ vs. $S_{90} / S_{140}$ & $S_{65} / S_{140}$ vs. $S_{90} / S_{160}$ & 98.70 & 94.46 & 64.45 & 73.33 \\
\hline$S_{65} / S_{140}$ vs. $S_{90} / S_{140}$ & $S_{65} / S_{140}$ vs. $S_{140} / S_{160}$ & 97.21 & 91.83 & 37.36 & 61.79 \\
\hline$S_{65} / S_{140}$ vs. $S_{90} / S_{140}$ & $S_{65} / S_{160}$ vs. $S_{90} / S_{140}$ & 98.92 & 95.59 & 67.64 & 80.92 \\
\hline$S_{65} / S_{140}$ vs. $S_{90} / S_{140}$ & $S_{65} / S_{160}$ vs. $S_{90} / S_{160}$ & 97.39 & 76.27 & 14.96 & 50.44 \\
\hline$S_{65} / S_{140}$ vs. $S_{90} / S_{140}$ & $S_{65} / S_{160}$ vs. $S_{140} / S_{160}$ & 99.22 & 95.37 & 72.34 & 80.88 \\
\hline$S_{65} / S_{140}$ vs. $S_{90} / S_{140}$ & $S_{90} / S_{140}$ vs. $S_{90} / S_{160}$ & 98.81 & 94.64 & 65.97 & 78.26 \\
\hline$S_{65} / S_{140}$ vs. $S_{90} / S_{140}$ & $S_{90} / S_{140}$ vs. $S_{140} / S_{160}$ & 99.01 & 95.56 & 71.13 & 81.48 \\
\hline$S_{65} / S_{140}$ vs. $S_{90} / S_{140}$ & $S_{90} / S_{160}$ vs. $S_{140} / S_{160}$ & 98.80 & 95.22 & 67.11 & 79.70 \\
\hline$S_{65} / S_{140}$ vs. $S_{90} / S_{160}$ & $S_{65} / S_{140}$ vs. $S_{140} / S_{160}$ & 98.31 & 97.19 & 69.21 & 81.72 \\
\hline$S_{65} / S_{140}$ vs. $S_{90} / S_{160}$ & $S_{65} / S_{160}$ vs. $S_{90} / S_{140}$ & 99.34 & 97.82 & 84.83 & 87.13 \\
\hline$S_{65} / S_{140}$ vs. $S_{90} / S_{160}$ & $S_{65} / S_{160}$ vs. $S_{90} / S_{160}$ & 97.89 & 78.88 & 48.36 & 45.92 \\
\hline$S_{65} / S_{140}$ vs. $S_{90} / S_{160}$ & $S_{65} / S_{160}$ vs. $S_{140} / S_{160}$ & 99.20 & 97.30 & 79.11 & 84.91 \\
\hline$S_{65} / S_{140}$ vs. $S_{90} / S_{160}$ & $S_{90} / S_{140}$ vs. $S_{90} / S_{160}$ & 98.86 & 96.27 & 74.80 & 79.63 \\
\hline$S_{65} / S_{140}$ vs. $S_{90} / S_{160}$ & $S_{90} / S_{140}$ vs. $S_{140} / S_{160}$ & 98.57 & 96.46 & 67.95 & 80.00 \\
\hline$S_{65} / S_{140}$ vs. $S_{90} / S_{160}$ & $S_{90} / S_{160}$ vs. $S_{140} / S_{160}$ & 98.77 & 96.81 & 73.82 & 81.55 \\
\hline$S_{65} / S_{140}$ vs. $S_{140} / S_{160}$ & $S_{65} / S_{160}$ vs. $S_{90} / S_{140}$ & 97.87 & 95.29 & 58.71 & 73.08 \\
\hline$S_{65} / S_{140}$ vs. $S_{140} / S_{160}$ & $S_{65} / S_{160}$ vs. $S_{90} / S_{160}$ & 98.48 & 79.36 & 68.84 & 48.24 \\
\hline$S_{65} / S_{140}$ vs. $S_{140} / S_{160}$ & $S_{65} / S_{160}$ vs. $S_{140} / S_{160}$ & 97.98 & 96.43 & 56.46 & 80.73 \\
\hline$S_{65} / S_{140}$ vs. $S_{140} / S_{160}$ & $S_{90} / S_{140}$ vs. $S_{90} / S_{160}$ & 97.31 & 94.04 & 49.54 & 68.47 \\
\hline$S_{65} / S_{140}$ vs. $S_{140} / S_{160}$ & $S_{90} / S_{140}$ vs. $S_{140} / S_{160}$ & 96.94 & 93.90 & 42.06 & 66.67 \\
\hline$S_{65} / S_{140}$ vs. $S_{140} / S_{160}$ & $S_{90} / S_{160}$ vs. $S_{140} / S_{160}$ & 97.17 & 94.26 & 48.54 & 67.92 \\
\hline$S_{65} / S_{160}$ vs. $S_{90} / S_{140}$ & $S_{65} / S_{160}$ vs. $S_{90} / S_{160}$ & 97.70 & 79.02 & 38.83 & 50.24 \\
\hline$S_{65} / S_{160}$ vs. $S_{90} / S_{140}$ & $S_{65} / S_{160}$ vs. $S_{140} / S_{160}$ & 98.96 & 97.07 & 70.34 & 85.47 \\
\hline$S_{65} / S_{160}$ vs. $S_{90} / S_{140}$ & $S_{90} / S_{140}$ vs. $S_{90} / S_{160}$ & 99.43 & 98.45 & 86.30 & 92.44 \\
\hline$S_{65} / S_{160}$ vs. $S_{90} / S_{140}$ & $S_{90} / S_{140}$ vs. $S_{140} / S_{160}$ & 99.09 & 98.28 & 77.88 & 91.38 \\
\hline$S_{65} / S_{160}$ vs. $S_{90} / S_{140}$ & $S_{90} / S_{160}$ vs. $S_{140} / S_{160}$ & 99.29 & 98.97 & 83.71 & 94.74 \\
\hline$S_{65} / S_{160}$ vs. $S_{90} / S_{160}$ & $S_{65} / S_{160}$ vs. $S_{140} / S_{160}$ & 98.01 & 80.25 & 38.66 & 54.72 \\
\hline$S_{65} / S_{160}$ vs. $S_{90} / S_{160}$ & $S_{90} / S_{140}$ vs. $S_{90} / S_{160}$ & 97.28 & 79.34 & 30.43 & 53.27 \\
\hline$S_{65} / S_{160}$ vs. $S_{90} / S_{160}$ & $S_{90} / S_{140}$ vs. $S_{140} / S_{160}$ & 96.89 & 78.03 & 19.50 & 49.29 \\
\hline$S_{65} / S_{160}$ vs. $S_{90} / S_{160}$ & $S_{90} / S_{160}$ vs. $S_{140} / S_{160}$ & 97.02 & 78.53 & 26.87 & 49.76 \\
\hline$S_{65} / S_{160}$ vs. $S_{140} / S_{160}$ & $S_{90} / S_{140}$ vs. $S_{90} / S_{160}$ & 98.65 & 95.47 & 63.37 & 79.03 \\
\hline$S_{65} / S_{160}$ vs $S_{140} / S_{160}$ & $S_{90} / S_{140}$ vs. $S_{140} / S_{160}$ & 98.63 & 95.67 & 62.21 & 79.34 \\
\hline$S_{65} / S_{160}$ vs. $S_{140} / S_{160}$ & $S_{90} / S_{160}$ vs. $S_{140} / S_{160}$ & 98.57 & 96.03 & 62.66 & 80.67 \\
\hline$S_{90} / S_{140}$ vs. $S_{90} / S_{160}$ & $S_{90} / S_{140}$ vs. $S_{140} / S_{160}$ & 99.51 & 98.78 & 88.64 & 94.31 \\
\hline$S_{90} / S_{140}$ vs. $S_{90} / S_{160}$ & $S_{90} / S_{160}$ vs. $S_{140} / S_{160}$ & 99.74 & 98.79 & 94.31 & 94.21 \\
\hline$S_{90} / S_{140}$ vs. $S_{140} / S_{160}$ & $S_{90} / S_{160}$ vs. $S_{140} / S_{160}$ & 99.67 & 99.31 & 92.60 & 96.61 \\
\hline
\end{tabular}

Objects for which no counterpart was found (there are only 22 of them) are also distributed in both parts of diagrams, and more than $30 \%$ of them are located in the "stellar" part of the diagrams. Their identity will be investigated in future works.

\section{Conclusions}

To separate stars and galaxies in the FIR AKARI all-sky survey data, we have selected a sample with the complete color information available in the low extinction regions of the sky and constructed color-color plots for these data. We looked for the method of separating stars and galaxies using the color information. For this purpose, we performed an extensive search for the counterparts of these selected all-sky survey objects in the NED and SIMBAD databases. Our conclusions are as follows.
1. The far infrared flux-color diagrams show two clear branches of sources. One of them is populated almost only by stars, the second one is dominated by galaxies.

2. The far infrared color-color diagrams allow for a high quality star-galaxy separation. In all the combinations of colorcolor diagrams we can distinguish two separate clouds. It was shown that one of them contains in all cases more than $95 \%$ of galaxies and the other one in most cases consists in more than $80 \%$ of stars.

3. At a first glance the population of stars is divided into (roughly) two categories: a larger group occupies a separate area on the diagrams while the remaining smaller part resides in the "galaxy cloud". A more careful examination has revealed that stars occupying a separate area on the colorcolor diagram are mostly known bright stars. This group is dominated by pulsating variable stars, among them many 
Mira-type variables, and also contains a number of evolved stars. Branch II of stars, overlapping the galaxy cloud, includes stars discovered by the infrared surveys, bright stars known to have an infrared excess, like Vega, pulsating stars, a few $\mathrm{T}$ Tauri stars and faint red stars. We estimate that the percentage of galaxies intervening in the stellar part of the diagram is in each case less than $6 \%$, and the percentage of stars in the galaxy part of the diagram - in most cases - less than $20 \%$. A few exceptions can probably be attributed to the larger error in the flux estimation, in particular in the $N 160$ band. Obviously, since this analysis was made in the sky regions with low Galaxtic cirrus emission, all the detailed numbers shown above are applicable to the regions except the low Galactic latitude zone.

4. We conclude that the FIR color-color diagrams are a powerful tool for separating stars from galaxies in the AKARI FIS all-sky survey, and in other, similarly large FIR catalogs. With this simple method we can select more than $95 \%$ of galaxies, rejecting $80 \%$ of stars. A more detailed methodology will be the subject of future work.

Acknowledgements. First, we thank the anonymous referee for her/his careful reading and comments of the manuscript, which improved the clarity of the paper very much. This work is based on observations with AKARI, a JAXA project with the participation of ESA. This research has made use of the NASA/IPAC Extragalactic Database (NED), which is operated by the Jet Propulsion Laboratory, California Institute of Technology, under contract with the National Aeronautics and Space Administration, and the SIMBAD database, operated at the CDS, Strasbourg, France. We deeply thank Issei Yamamura for his huge effort to check the reliability of our stellar identifications and their classification. A.P. was financed by the research grant of the Polish Ministry of Science PBZ/MNiSW/07/2006/34A. TTT has been supported by the Program for Improvement of Research Environment for Young Researchers from Special Coordination Funds for Promoting Science and Technology, and the Grant-inAid for the Scientific Research Fund (20740105) commissioned by the Ministry of Education, Culture, Sports, Science and Technology (MEXT) of Japan. T.T.T. has been partially supported from the Grand-in-Aid for the Global COE Program "Quest for Fundamental Principles in the Universe: from Particles to the Solar System and the Cosmos" from the MEXT.

\section{References}

Buat, V., Takeuchi, T. T., Iglesias-Páramo, J., et al. 2007a, ApJS, 173, 404 Buat, V., Marcillac, D., Burgarella, D., et al. 2007b, A\&A, 469, 19

Buat, V., Boissier, S., Burgarella, D., et al. 2008, A\&A, 483, 107 Caputi, K., Lagache, G., Yan, L., et al. 2007, ApJ, 660, 97 Kawada, M., Baba, H., Barthel, P., et al. 2007, PASJ, 59, 389 Le Floc'h, E., Papovich, C., \& Dole, H. 2005, ApJ, 632, 169 Małek, K., Pollo, A., Takeuchi, T. T., et al. 2010, A\&A, 514, A11 Murakami, H., Baba, H., Barthel, P., et al. 2007, PASJ, 59, 369

Neugebauer, G., Habing, H. J., van Duinen, R., et al. 1984, ApJ, 278, L1 Onaka, T., Matsuhara, H., Wada, T., et al. 2007, PASJ, 59, S401 Reddy, N. A., Steidel, C. C., Pettini, M., et al. 2008, ApJS, 175, 48 Schlegel, D. J., Finkbeiner, D. P., \& Davis, M. 1998, ApJ, 500, 525 Takeuchi, T. T., Hirashita, H., Ohta, K., et al. 1999, PASP, 111, 288

Takeuchi, T. T., Buat, V., \& Burgarella, D. 2005, A\&A, 440, L17 Takeuchi, T. T., Buat, V., Heinis, S., et al. 2010, A\&A, 514, A4 Walker, H. J., Cohen, M., Volk, K., Wainscoat, R. J., \& Schwartz, D. E. 1989, AJ, 98, 2163

Yamamura, I., Fukuda, Y., \& Makiuti, S. 2008, AKARI/FIS All-Sky Survey Bright Source Catalogue Version $\beta$-1 Release Note (Rev. 1)

Yamamura, I., et al. 2009, in AKARI, a light to illuminate the misty Universe, ed. T. Onaka, G. White, T. Nakagawa, \& I. Yamamura, ASP Conf. Ser., in press 


\section{Appendix A: Other flux-color diagrams}
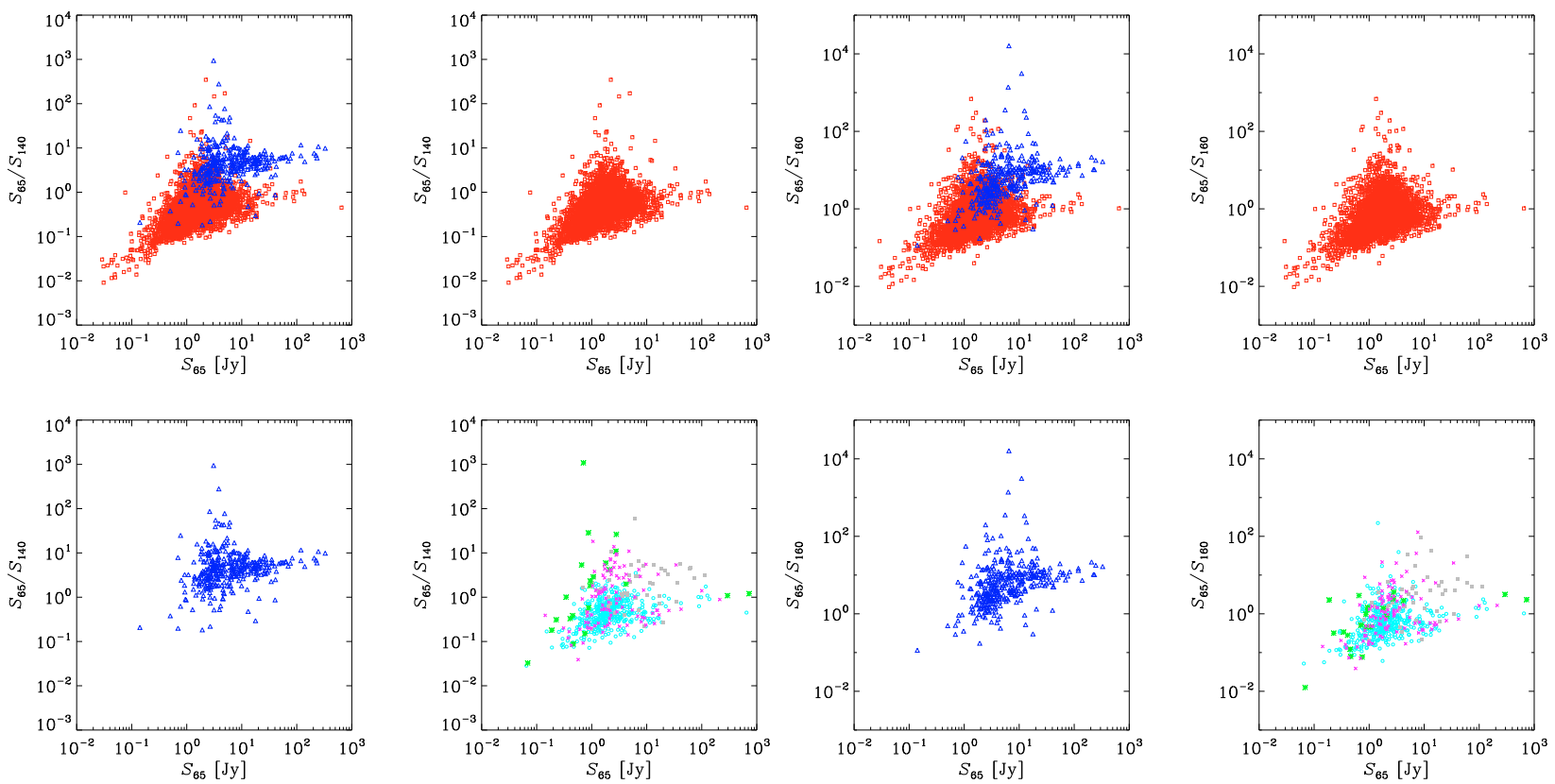

Fig. A.1. Same as Fig. 3 but for $S_{65}-S_{65} / S_{140}$ (four panels on the left side) and for $S_{65}-S_{65} / S_{160}$ (four panels on the right side).
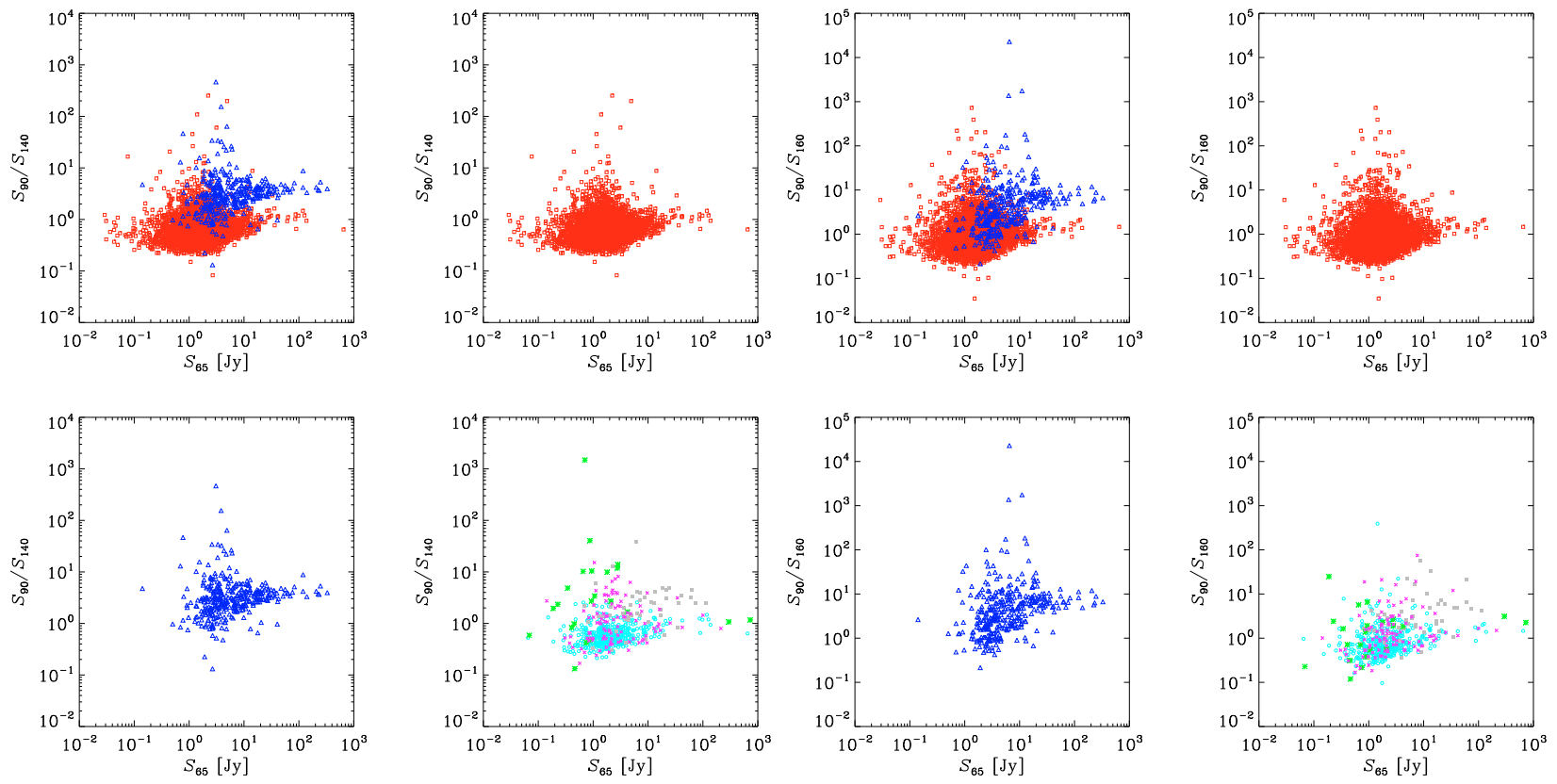

Fig. A.2. Same as Fig. 3 but for $S_{65}-S_{90} / S_{140}$ (four panels on the left side) and $S_{65}-S_{90} / S_{160}$ (four panels on the right side). 
A. Pollo et al.: Star-galaxy separation by far infrared colors
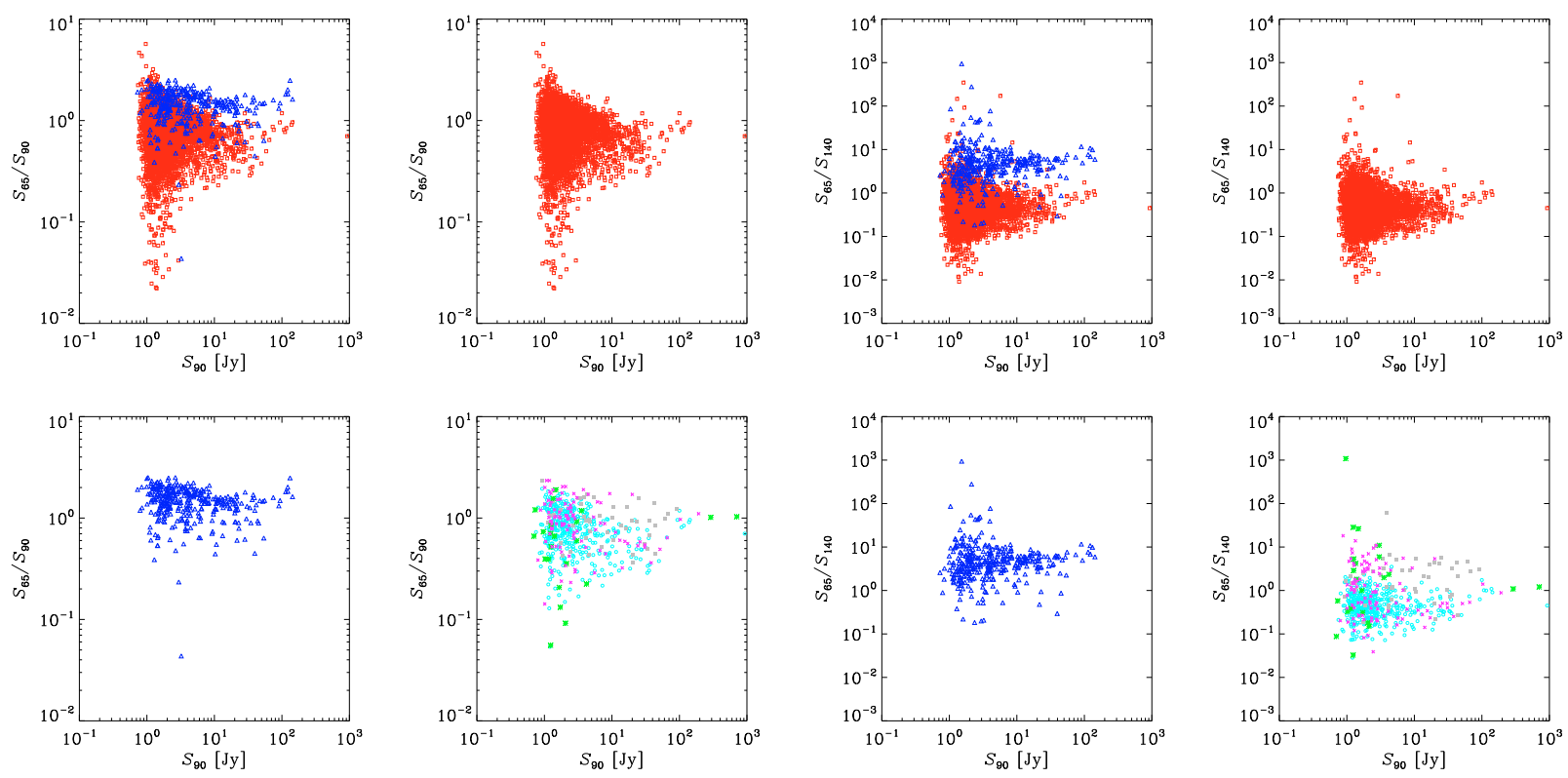

Fig. A.3. Same as Fig. 3 but for $S_{90}-S_{65} / S_{90}$ (four panels on the left side) and $S_{90}-S_{65} / S_{140}$ (four panels on the right side).
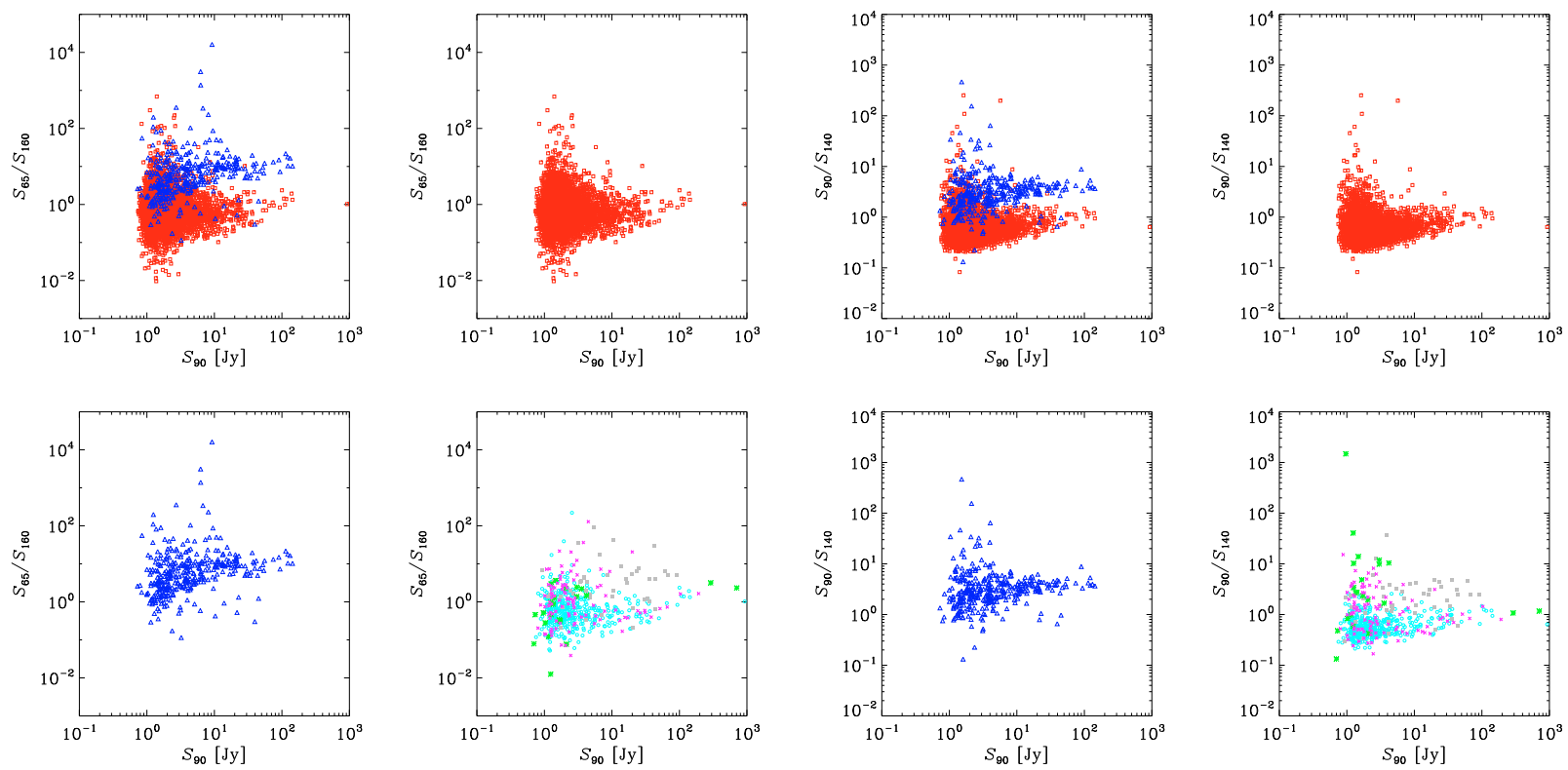

Fig. A.4. Same as Fig. 3 but for $S_{90}-S_{65} / S_{160}$ (four panels on the left side) and $S_{90}-S_{90} / S_{140}$ (four panels on the right side). 
A\&A 514, A3 (2010)
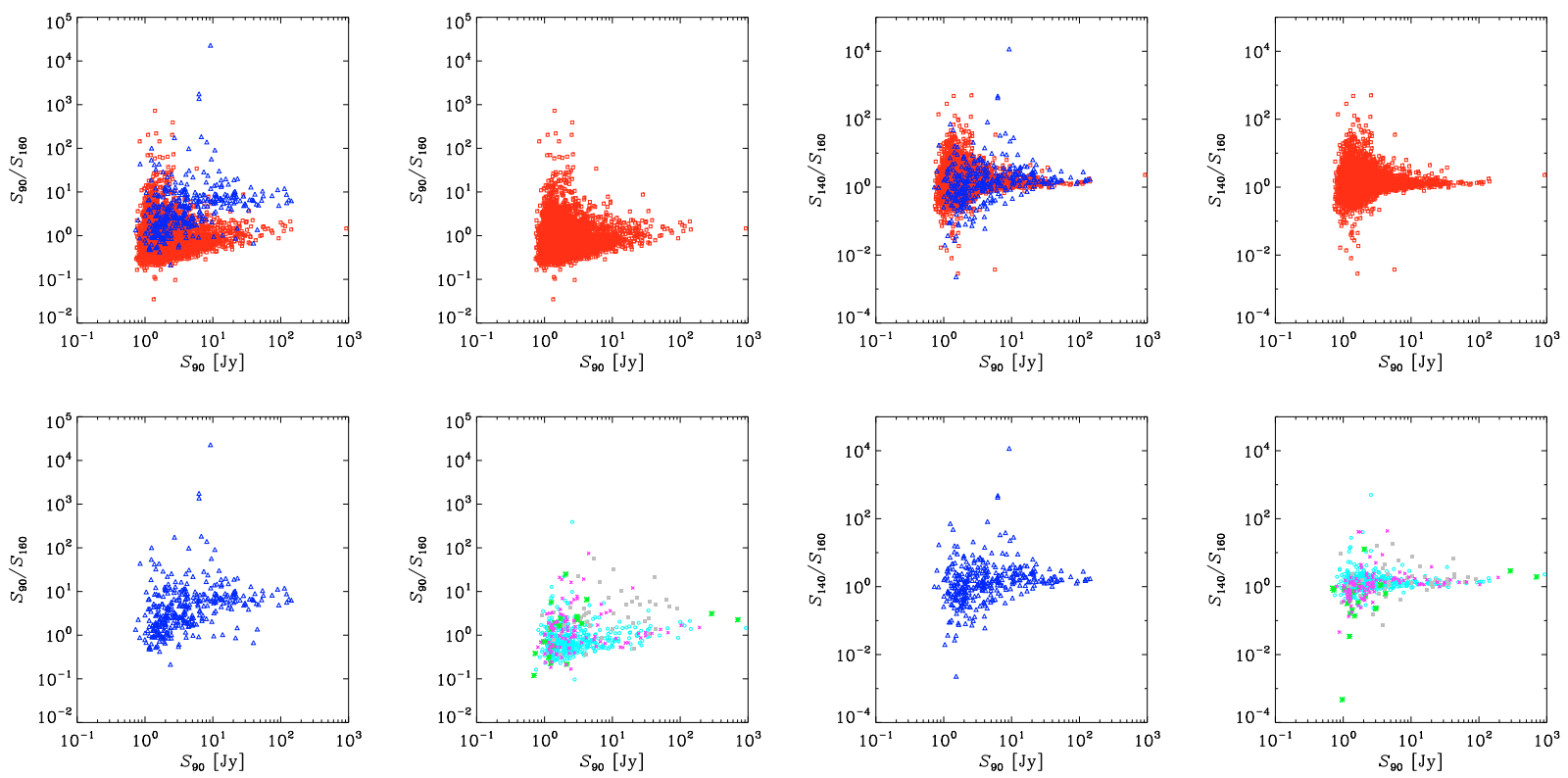

Fig. A.5. Same as Fig. 3 but for $S_{90}-S_{90} / S_{160}$ (four panels on the left side) and $S_{90}-S_{140} / S_{160}$ (four panels on the right side).
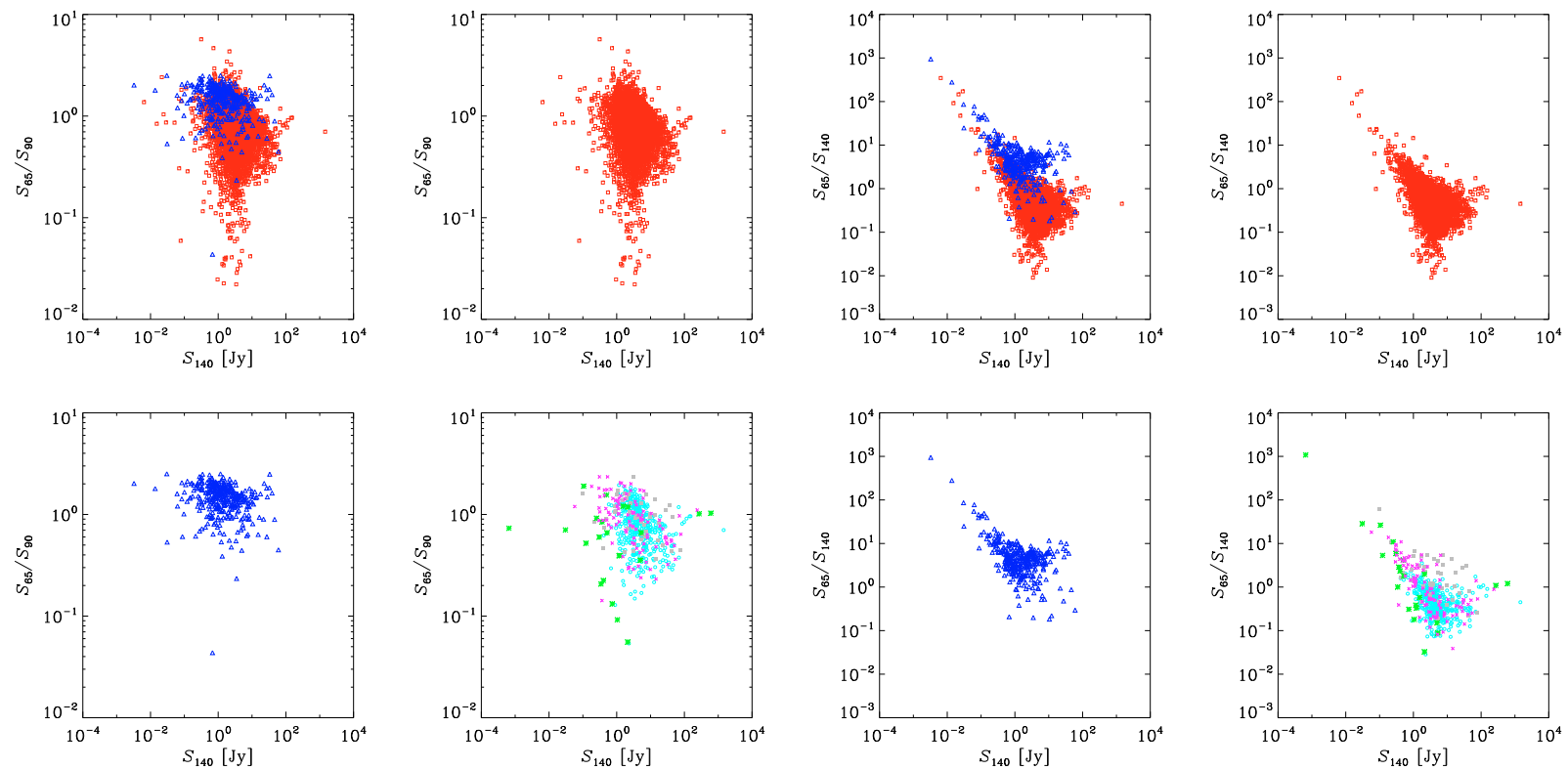

Fig. A.6. Same as Fig. 3 but for $S_{140}-S_{65} / S_{90}$ (four panels on the left side) and $S_{140}-S_{65} / S_{140}$ (four panels on the right side). 
A. Pollo et al.: Star-galaxy separation by far infrared colors
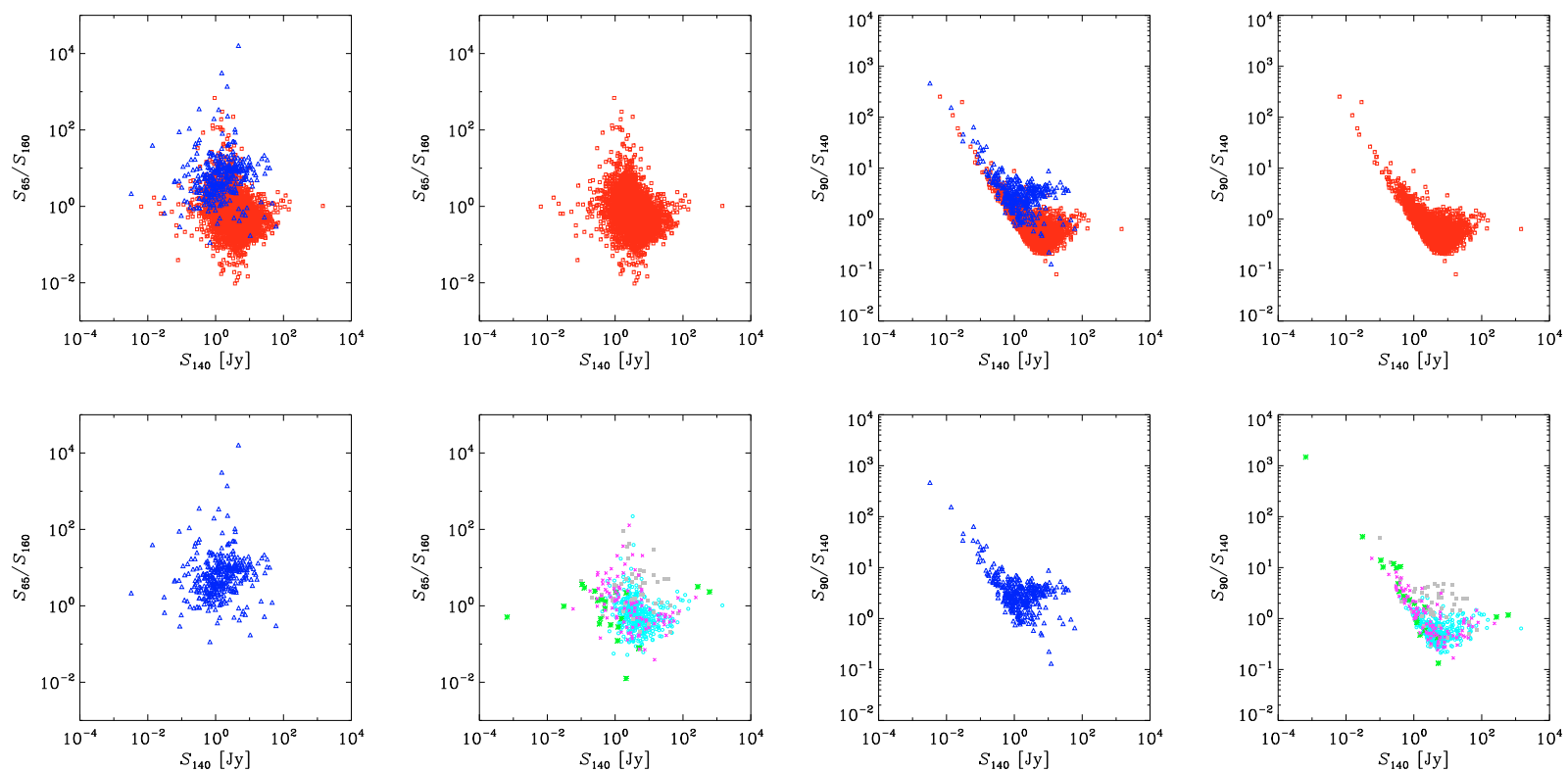

Fig. A.7. Same as Fig. 3 but for $S_{140}-S_{65} / S_{160}$ (four panels on the left side) and $S_{140}-S_{90} / S_{140}$ (four panels on the right side).
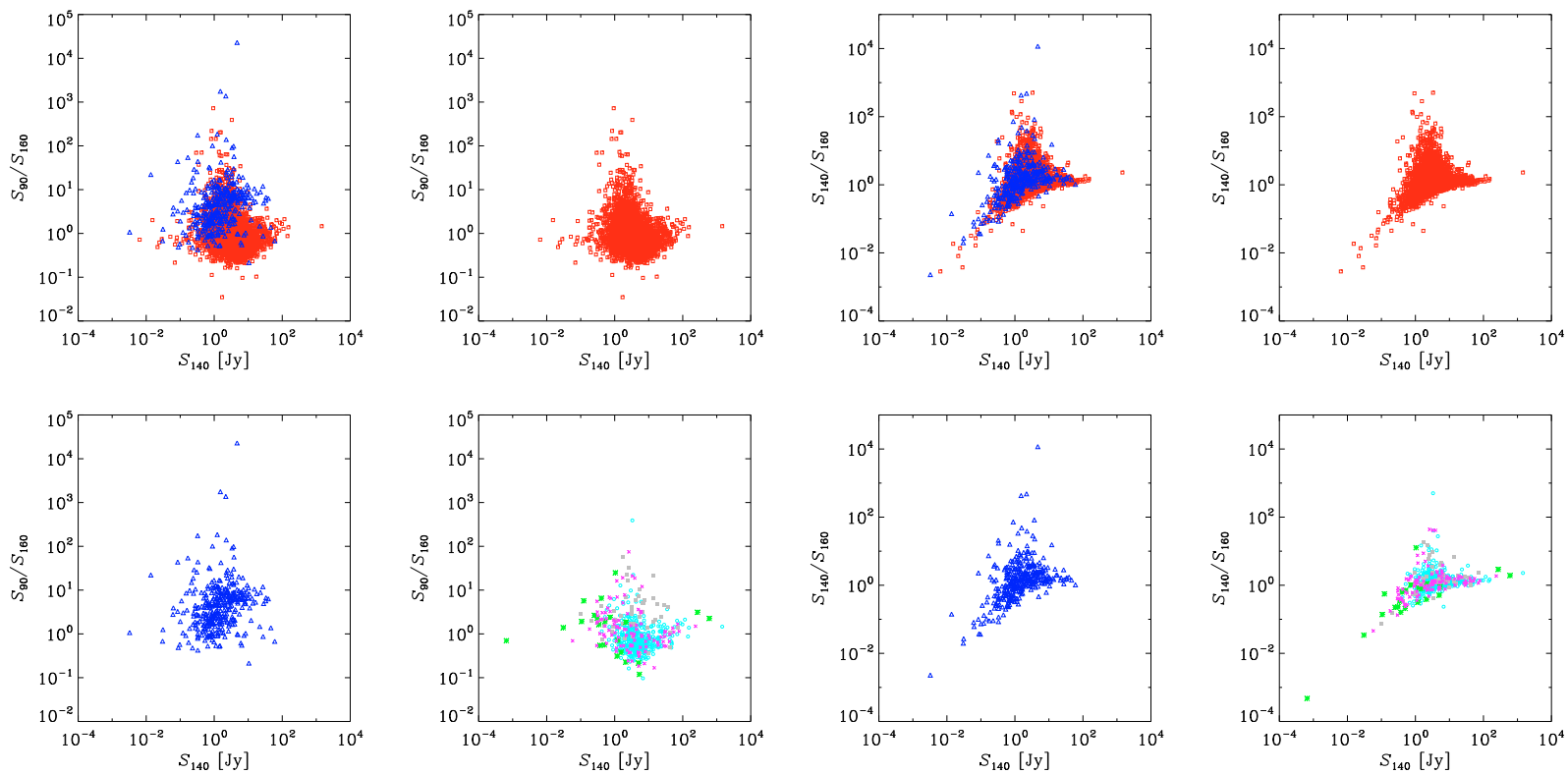

Fig. A.8. Same as Fig. 3 but for $S_{140}-S_{90} / S_{160}$ (four panels on the left side) and $S_{140}-S_{140} / S_{160}$ (four panels on the right side). 
A\&A 514, A3 (2010)
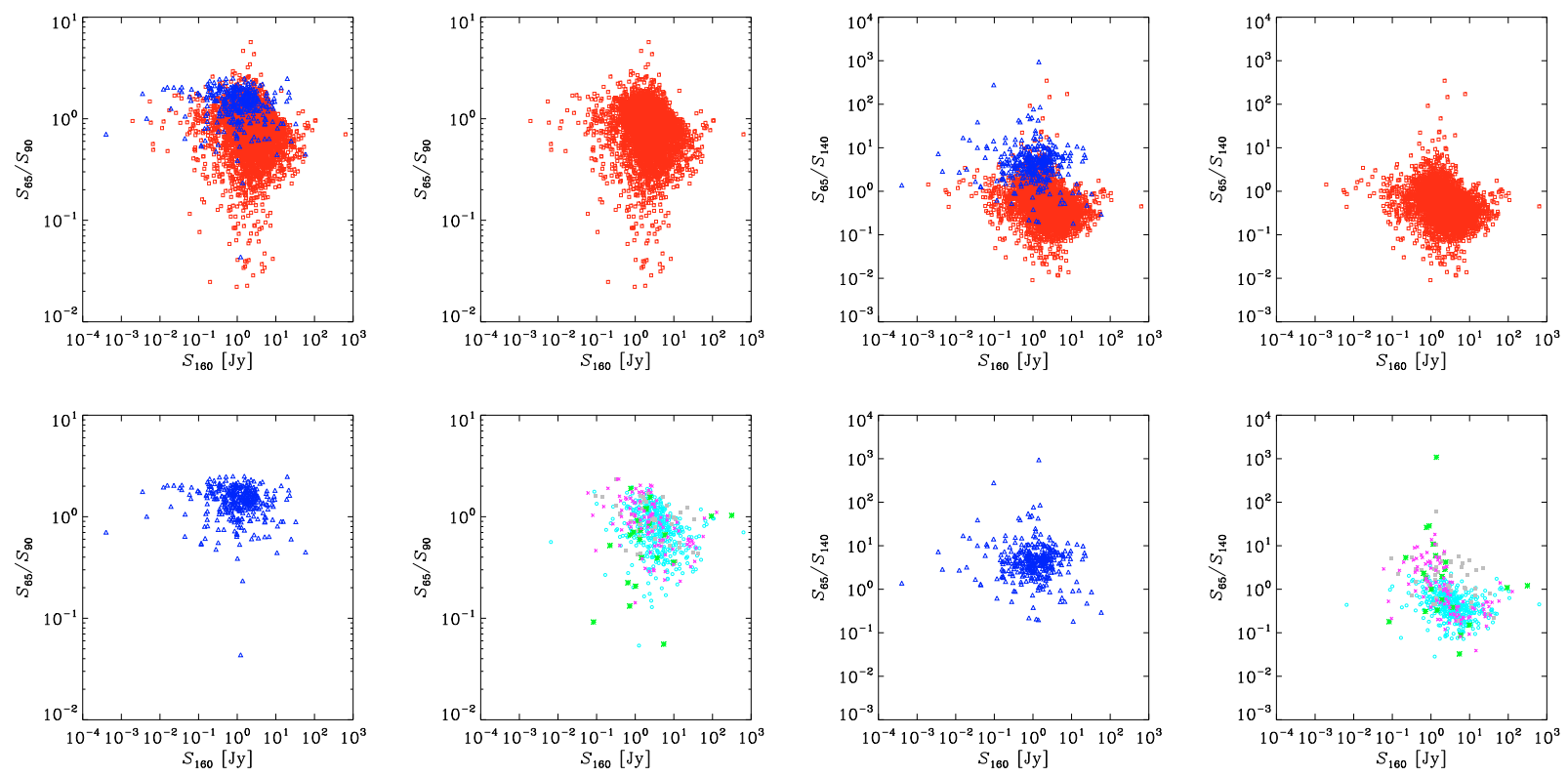

Fig. A.9. Same as Fig. 3 but for $S_{160}-S_{65} / S_{90}$ (four panels on the left side) $S_{160}-S_{65} / S_{140}$ (four panels on the right side).
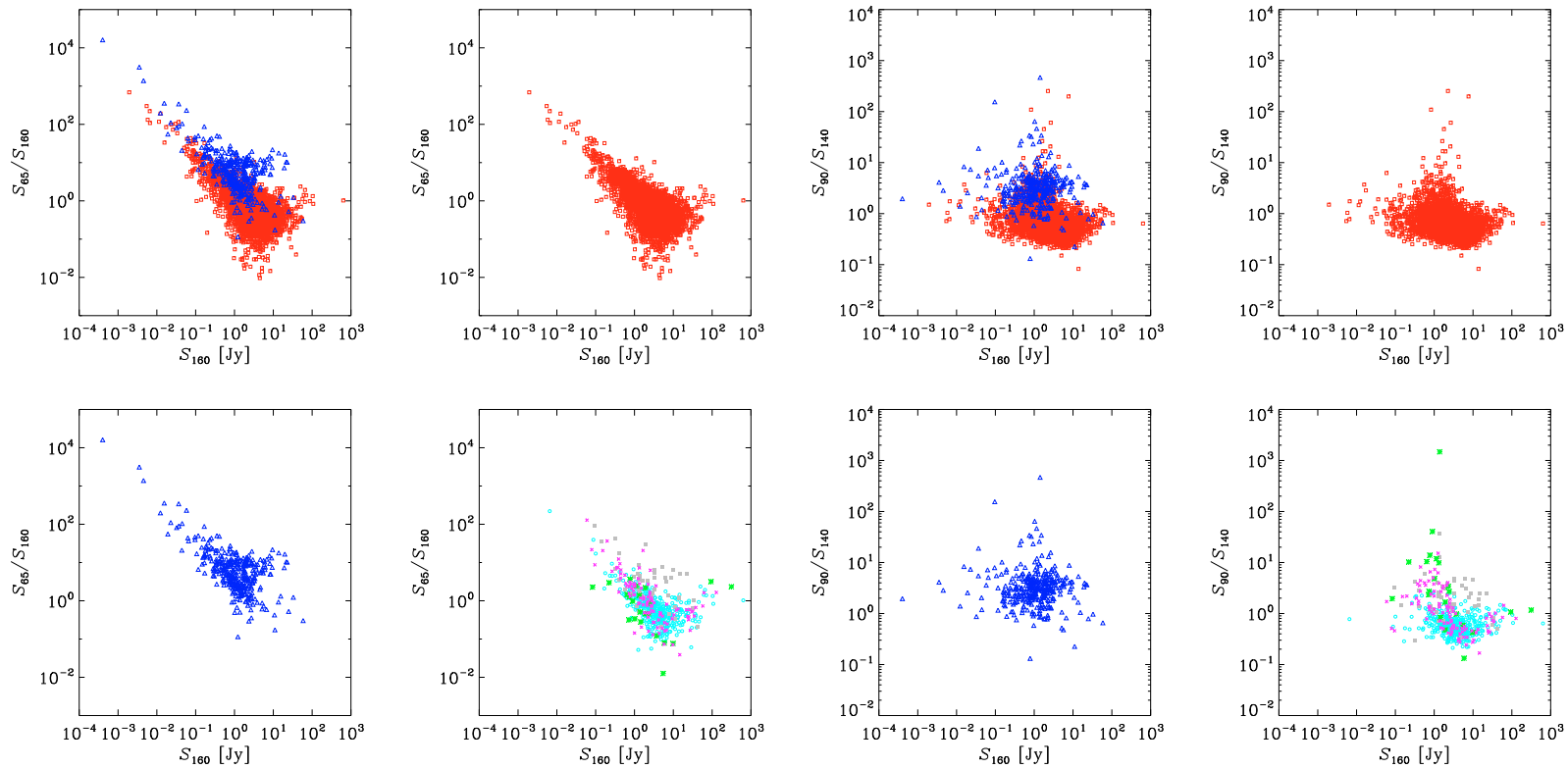

Fig. A.10. Same as Fig. 3 but for $S_{160}-S_{65} / S_{160}$ (four panels on the left side) and $S_{160}-S_{90} / S_{140}$ (four panels on the right side). 
A. Pollo et al.: Star-galaxy separation by far infrared colors
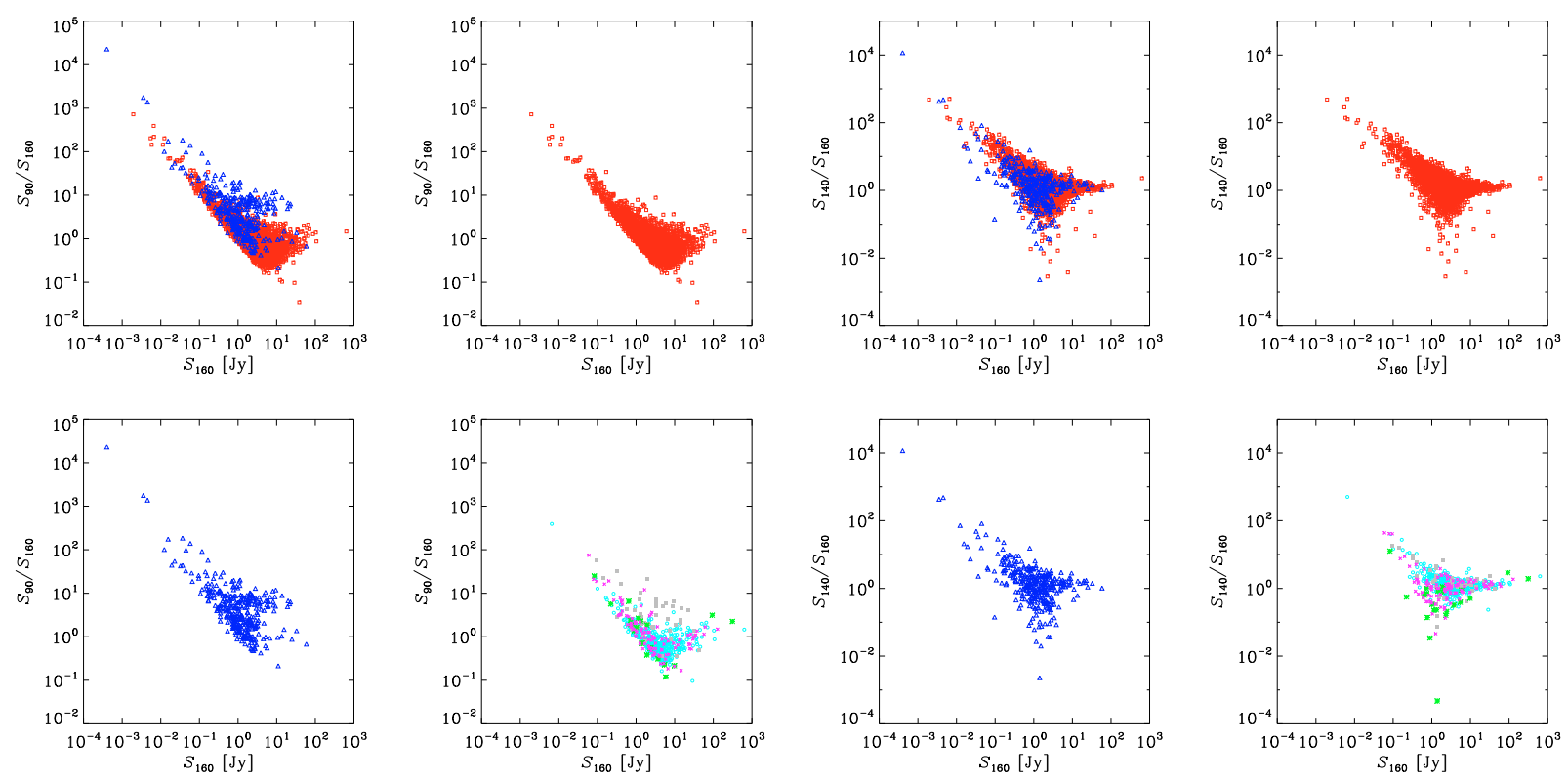

Fig. A.11. Same as Fig. 3 but for $S_{160}-S_{90} / S_{160}$ (four panels on the left side) and $S_{160}-S_{140} / S_{160}$ (four panels on the right side).

\section{Appendix B: Other color-color diagrams}
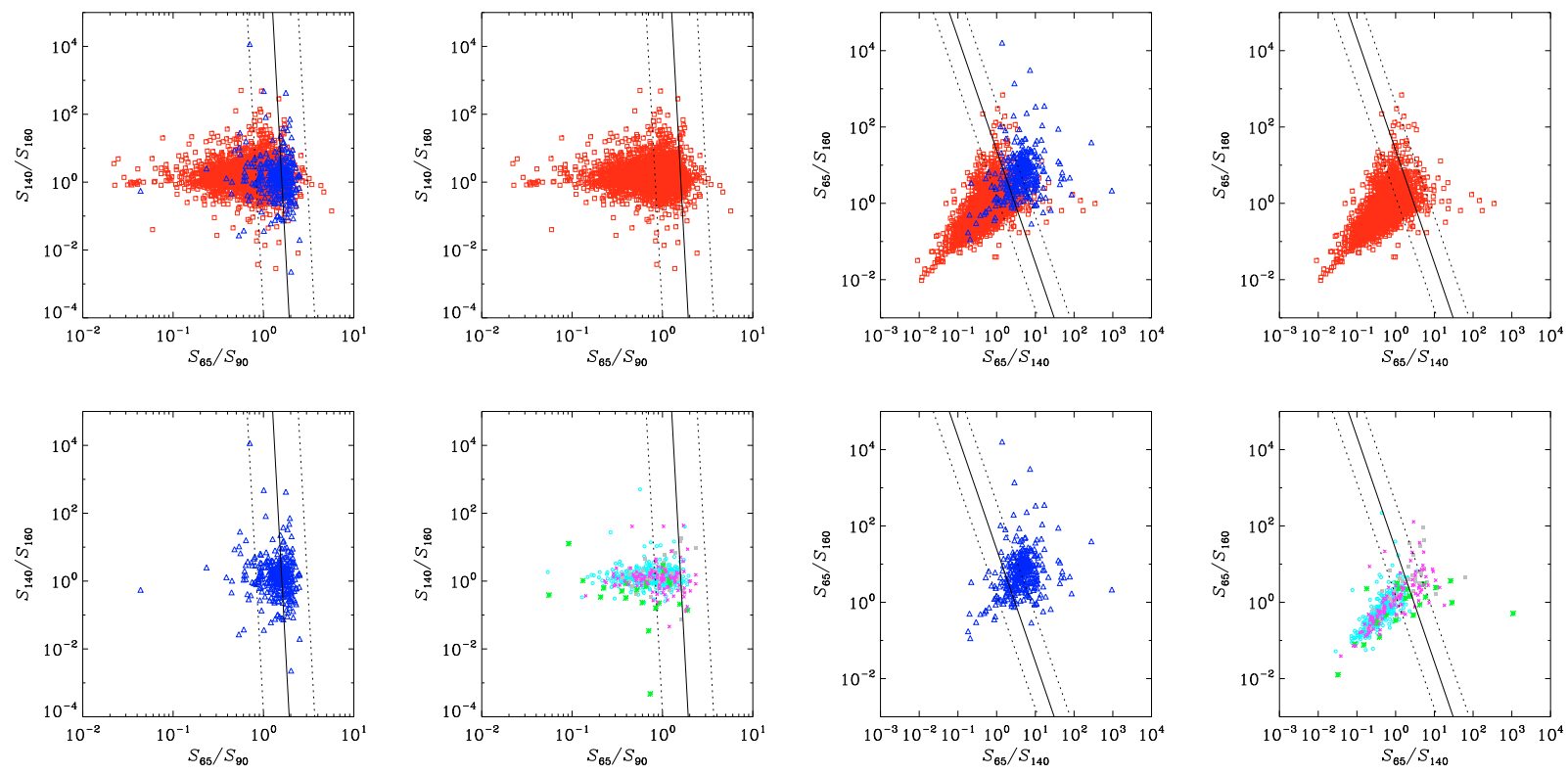

Fig. B.1. Same as Fig. 5 but for $S_{140} / S_{160}-S_{65} / S_{90}$ (four panels on the left side) and $S_{65} / S_{160}-S_{65} / S_{140}$ (four panels on the right side). 
A\&A 514, A3 (2010)
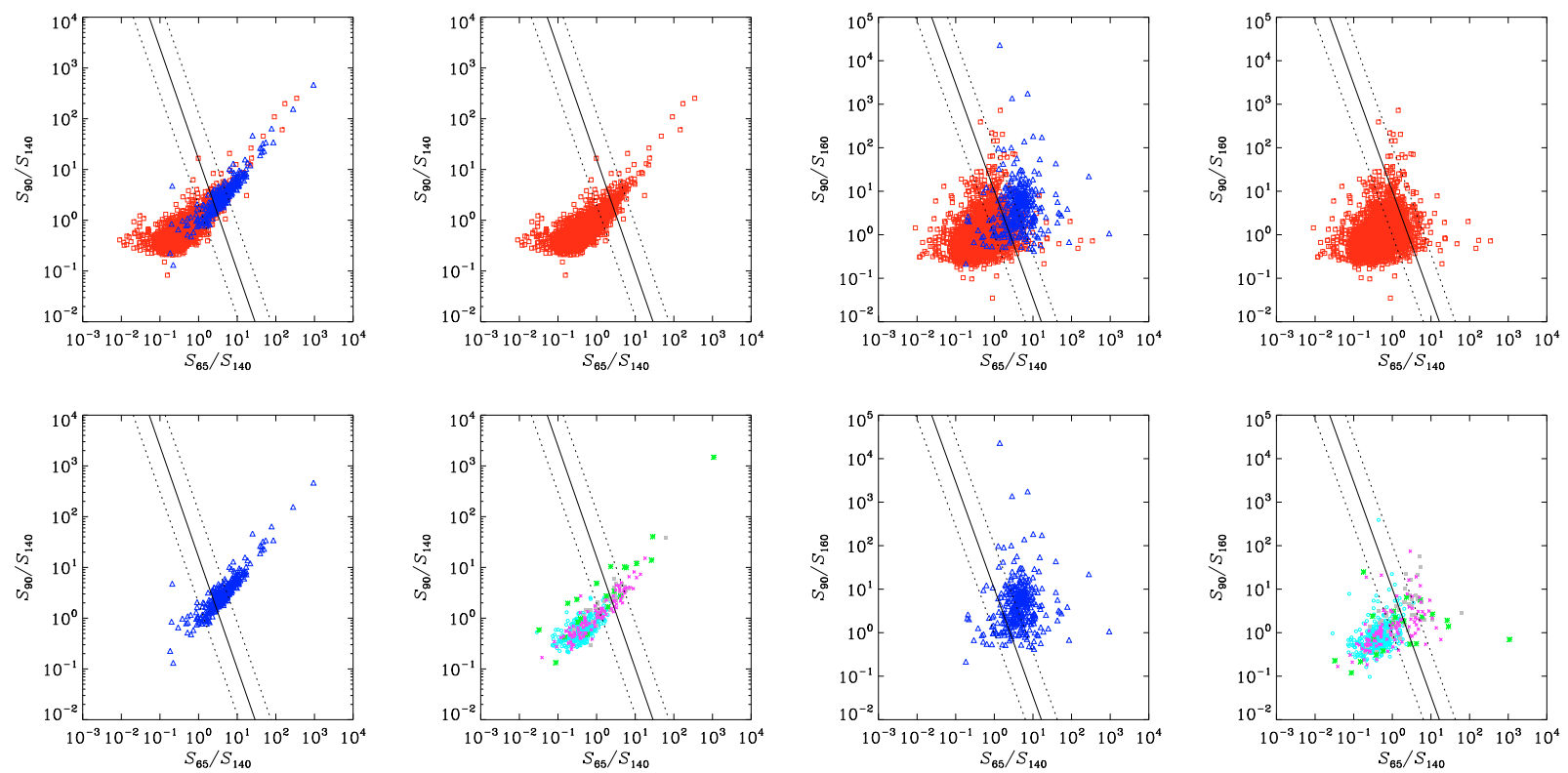

Fig. B.2. Same as Fig. 5 but for $S_{90} / S_{140}-S_{65} / S_{140}$ (four panels on the left side) and $S_{90} / S_{160}-S_{65} / S_{140}$ (four panels on the right side).
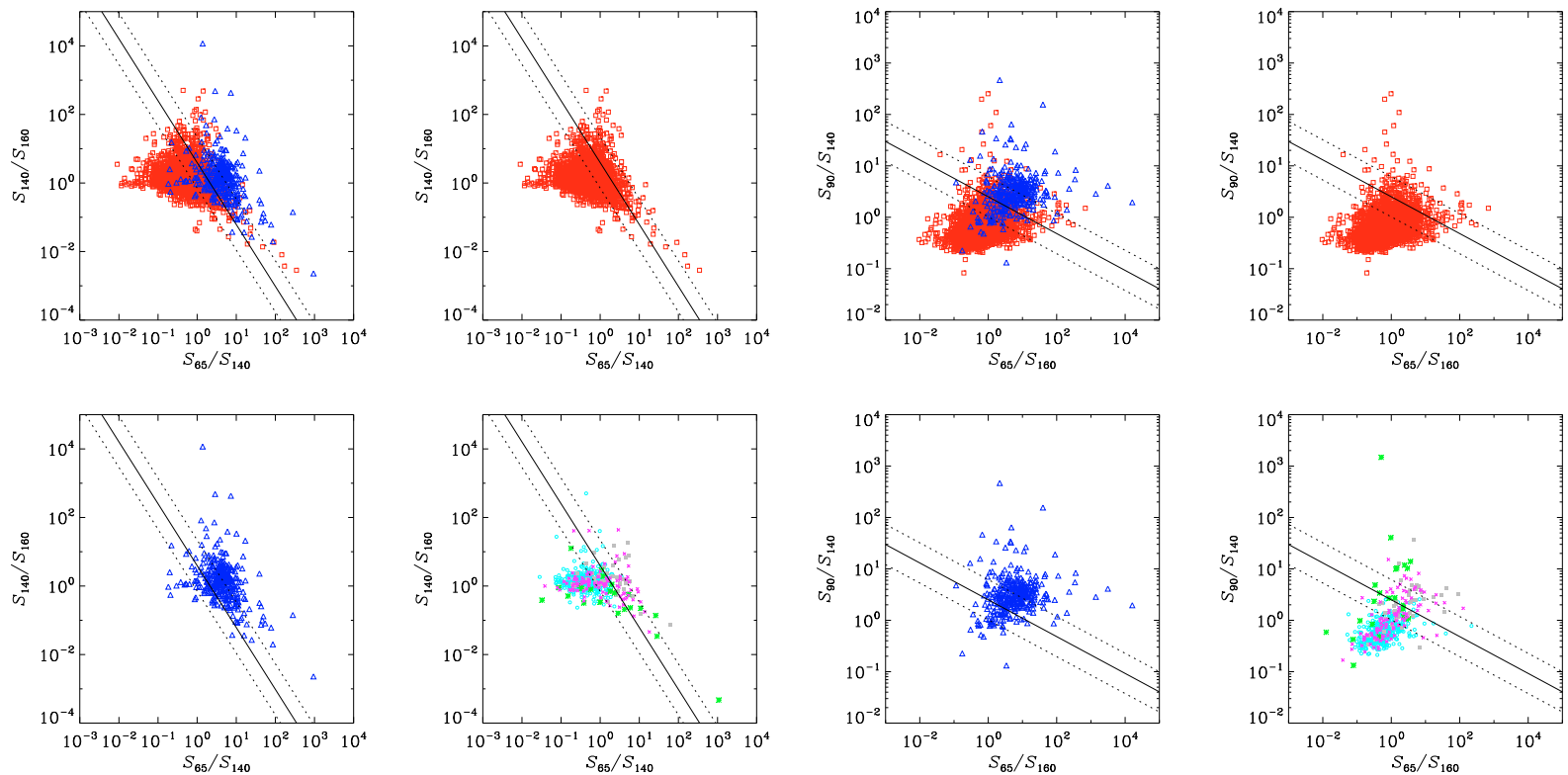

Fig. B.3. Same as Fig. 5 but for $S_{140} / S_{160}-S_{65} / S_{140}$ (four panels on the left side) and $S_{90} / S_{140}-S_{65} / S_{160}$ (four panels on the right side). 
A. Pollo et al.: Star-galaxy separation by far infrared colors
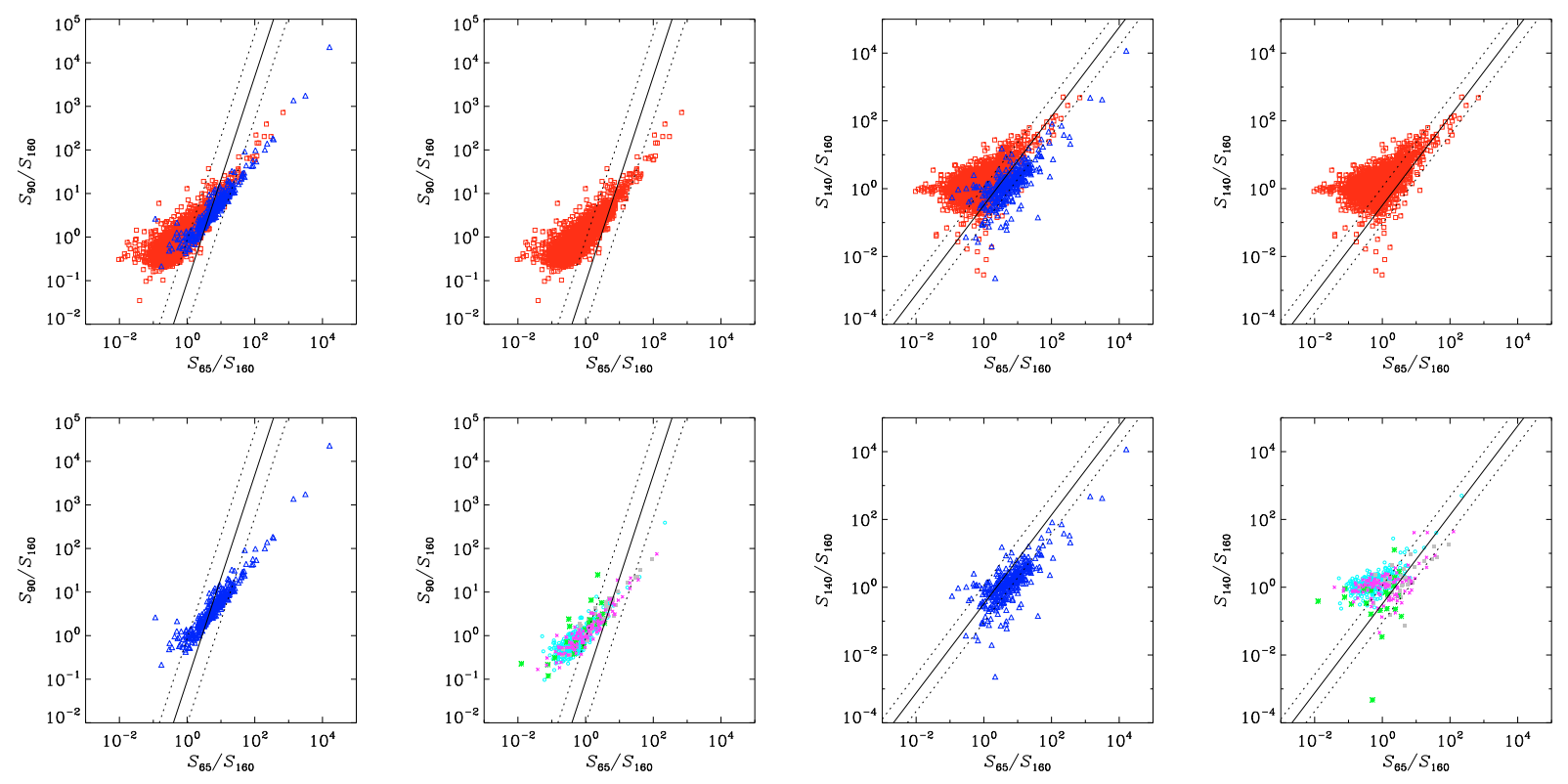

Fig. B.4. Same as Fig. 5 but for $S_{90} / S_{160}-S_{65} / S_{160}$ (four panels on the left side) and $S_{140} / S_{160}-S_{65} / S_{160}$ (four panels on the right side).
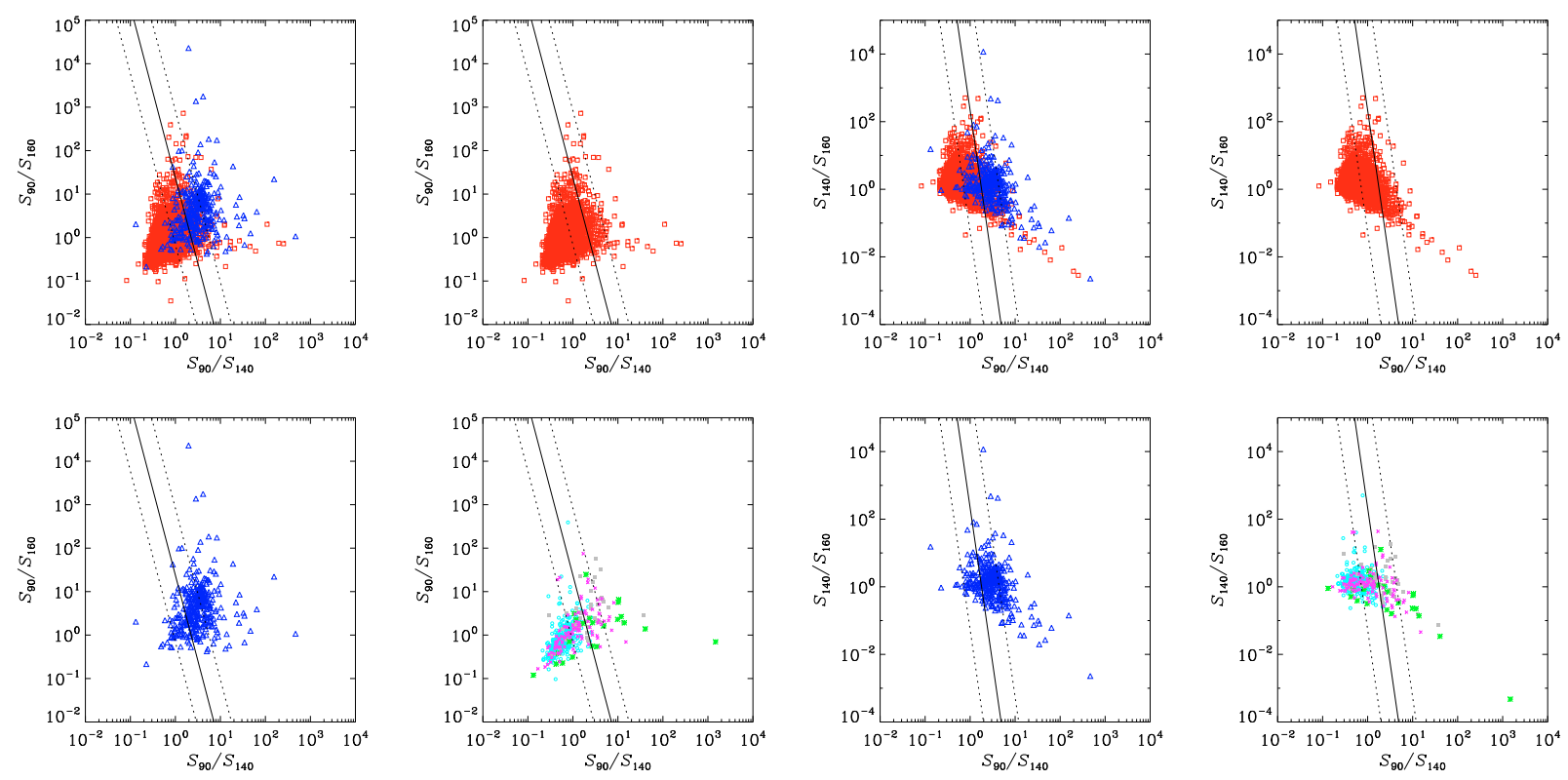

Fig. B.5. Same as Fig. 5 but for $S_{90} / S_{160}-S_{90} / S_{140}$ (four panels on the left side) and $S_{140} / S_{160}-S_{90} / S_{140}$ (four panels on the right side). 
A\&A 514, A3 (2010)
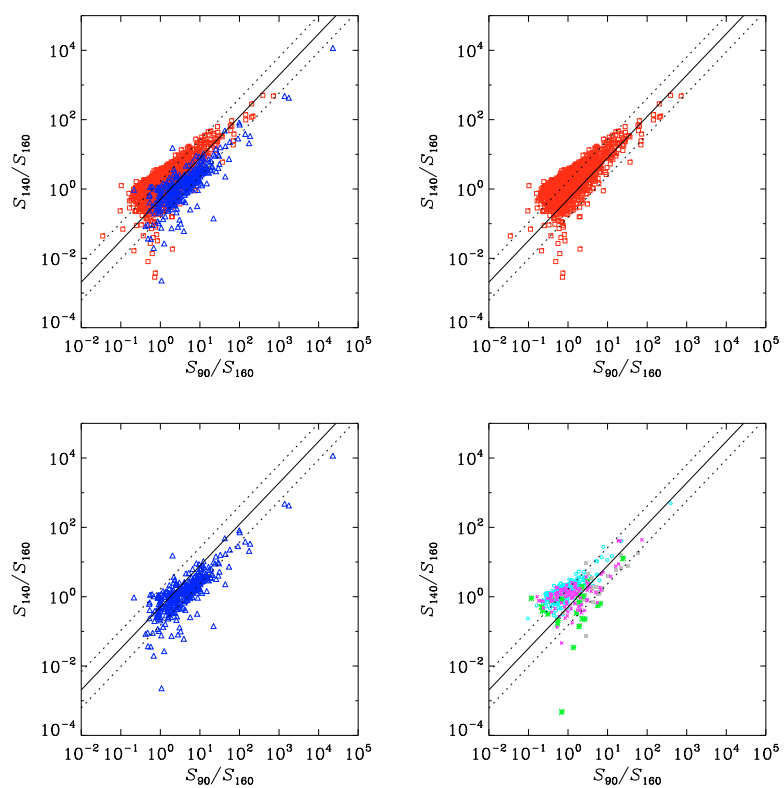

Fig. B.6. Same as Fig. 5 but for $S_{140} / S_{160}-S_{90} / S_{160}$. 